\title{
Complex geometry and supergeometry
}

\author{
Eric D'Hoker and D.H. Phong
}

\begin{abstract}
Complex geometry and supergeometry are closely entertwined in superstring perturbation theory, since perturbative superstring amplitudes are formulated in terms of supergeometry, and yet should reduce to integrals of holomorphic forms on the moduli space of punctured Riemann surfaces. The presence of supermoduli has been a major obstacle for a long time in carrying out this program. Recently, this obstacle has been overcome at genus 2 , which is the first loop order where it appears in all amplitudes. An important ingredient is a better understanding of the relation between geometry and supergeometry, and between holomorphicity and superholomorphicity. This talk provides a survey of these developments and a brief discussion of the directions for further investigation.
\end{abstract}

\section{Introduction}

String theory is a theory of random surfaces. Perturbative scattering amplitudes of string states are sums over the fluctuating worldsheets spanned by evolving strings. Conformal invariance reduces these sums to sums over only conformally distinct worldsheets. Thus, perturbatively, string scattering amplitudes should be given by series of integrals over the moduli space $\mathcal{M}_{h}$ of Riemann surfaces of genus $h \geq 0$.

An early major success of superstring theory was the explicit oneloop $(h=1)$ amplitudes obtained by Green and Schwarz [1] for the superstring and by Gross et al. [2] for the heterotic string. However, the general loop order $h$ has remained intractable to this day. This is due to a fundamental geometric difficulty beginning at $h=2$, which is the occurrence of $2 h-2$ odd supermoduli inherent to the Neveu-SchwarzRamond formulation of the superstring $[3,4]$.

Lecture at "Current Developments in Mathematics", November 2005, Cambridge, MA. Research supported in part by National Science Foundation grants PHY-01-40151, DMS-02-45371, PHY-0456200, and by the KITP (Santa Barbara) under NSF grant PHY-99-07949. 
In the NSR formulation, the sums over fluctuating worldsheets for the superstring are realized by integrating over all supergeometries $\left(g_{m n}, \chi_{m}{ }^{\alpha}\right)$ instead of over all geometries $g_{m n}$, where $g_{m n}$ are metrics on a fixed smooth surface $\Sigma$ and $\chi_{m}{ }^{\alpha}$ are gravitino fields on $\Sigma$. The standard Faddeev-Popov gauge fixing procedure reduces these sums to integrals over the supermoduli space $s \mathcal{M}_{h}$ of inequivalent supergeometries instead of integrals over the moduli space $\mathcal{M}_{h}$ of inequivalent geometries. The space $s \mathcal{M}_{h}$ is a $(3 h-3 \mid 2 h-2)$ superspace, and the $2 h-2$ odd supermoduli have to be integrated out in order to arrive at the desired integrals over $\mathcal{M}_{h}$. This is a new step beyond the standard gauge fixing procedures of quantum field theory. It is not made any easier by our insufficient understanding of the interplay between local supersymmetry and the complex structures of Riemann surfaces and their moduli space.

Recently, however, the supermoduli problem has been overcome for the case of genus $h=2$ and even spin structures $[\mathbf{5}, \mathbf{6}, \mathbf{7}, \mathbf{8}, \mathbf{9}, \mathbf{1 0}]$, which is the first loop order where it appears in all amplitudes. The progress is based partly on an improved understanding of the interplay between worldsheet supersymmetry and complex structures. In particular, at genus $h=2$ and even spin structures, we have now:

- A gauge-fixing procedure which reduces the sums over fluctuating worldsheets in superstring theory to well-defined integrals over the moduli space $\mathcal{M}_{2}$ of Riemann surfaces of genus $h=2$, in $[\mathbf{5}, \mathbf{6}]$.

- These integrals are independent of the choice of gauge slices $[\mathbf{6}, \mathbf{7}]$. As pointed out in $[\mathbf{1 1}, \mathbf{1 2}]$, gauge slice independence is a crucial requirement which was not satisfied by the Ansätze for superstring amplitudes proposed in the past.

- Underlying this gauge slice independence is the remarkable fact that gauge slice changes produce global forms which are de Rham-exact in all insertion points, point by point over moduli space [9].

- The integrands of the superstring scattering amplitudes are hermitian pairings of holomorphic forms of maximal rank on the moduli space of Riemann surfaces with punctures. Holomorphicity is a particularly important property for string theory, indispensable for example in the construction of heterotic strings. The holomorphicity of the superstring integrand is recovered from superholomorphicity by extracting a term which is Dolbeault exact in one insertion point and de Rham exact in the remaining insertion points $[\mathbf{9}, \mathbf{1 0}]$.

- The measure on the moduli space of Riemann surfaces for each spin structure $\delta$ has been evaluated in terms of $\vartheta$-constants [8]. It is given by a modular covariant form $\Xi_{6}[\delta](\Omega)$ of weight 6 , which may be interesting in its own right. 
- Using the above measure, the 0-, 1-, 2-, and 3-point scattering amplitudes for massless NS states have been evaluated and found to vanish identically, both for the type II and heterotic superstrings [10]. These results provide a proof, from first principles and to two-loop order, of "non-renormalization theorems" which had been conjectured [13] on the basis of space-time supersymmetry.

- The first non-vanishing two-loop amplitude, namely the scattering amplitude of 4 massless NS bosons, has also been evaluated explicitly, for the first time in a gauge slice independent formalism for both the type II and heterotic superstrings [10]. Its surprisingly simple form may give a clue to the 4-point function for higher genus.

- The two-loop string corrections to certain terms in the low energy effective action for both type II and heterotic superstrings have been computed precisely $[\mathbf{1 0}, \mathbf{1 4}]$. In particular, for the type II theories, the $\mathcal{R}^{4}$ correction is absent, while for the heterotic theories, $\operatorname{the} \operatorname{tr} F^{4}$, $\operatorname{tr} F^{2} \operatorname{tr} F^{2}, \mathcal{R}^{2} \operatorname{tr} F^{2}$, and $\mathcal{R}^{4}$ corrections are all absent, thus confirming predictions made on the basis of $S$-duality in type IIB theory and space-time supersymmetry. The non-vanishing two-loop correction to the $D^{4} \mathcal{R}^{4}$ term in Type IIB theory has been matched precisely against earlier predictions made on the basis of $S$-duality and space-time supersymmetry, by Green and collaborators (joint work with M. Gutperle $[14])$.

- The issue of whether the two-loop cosmological constant vanishes point by point on moduli space for certain $Z_{2}$-orbifold models proposed by Kachru, Kumar, and Silverstein [15] has been resolved. These are models with broken supersymmetry but vanishing one-loop cosmological constant. There had been hope that the two-loop cosmological constant would also vanish, but we find that this is not the case (joint work with K. Aoki $[\mathbf{1 6}])$.

The goal of this lecture is to provide a brief survey of these developments, with emphasis on the geometric aspects. Superstring perturbation theory has received sustained attention over the years $[\mathbf{1 1}$, $17,18,19,20,21,22,23]$, and has motivated many mathematical developments (see e.g., $[\mathbf{2 4}, \mathbf{2 5}]$ and references therein). The formulation of superstring perturbation theory adopted here is the NSR formulation. Though complicated by the presence of supermoduli at higher loop level, and by the necessity to perform summations over spin structures, the NSR formulation is based on the worldsheet action of 2-d supergravity whose quantization is well-understood, and on firm ground. The Green-Schwarz formulation has the advantage of manifest space-time supersymmetry and no need for supermoduli and spin structures, but its systematic quantization beyond 1-loop order has not yet been achieved, in part because of the presence of delicate second class 
constraints. Perhaps more promising is the pure spinor formulation of Berkovits [26], which circumvents the second class constraints, and permits direct quantization. Yet, it is unclear whether this formulation possesses an ungauge-fixed action, as is customarily used for a starting point. (See however [27].) ${ }^{1}$

Acknowledgements. The authors are grateful to K. Aoki and M. Gutperle for collaboration on parts of this project. They would like to thank Costas Bachas, Gerard van der Geer, Michael Green, Sam Grushevky, Boris Pioline, Jacob Sturm, Tomasz Taylor, Richard Wentworth, and Edward Witten for useful conversations, correspondences, and references.

\section{Description of the Main Results}

In this section, we provide a fuller description of the main results, leaving a sketch of their derivation to the next section.

Our main goal is a systematic method for the evaluation of scattering amplitudes at genus 2 of $N$ massless bosonic states in superstring theory. This can be viewed as the string analogue of the Feynman rules of quantum field theory, with the two-loop diagram being a unique topological surface $\Sigma$, and Feynman parameters given by moduli. We consider both the type II superstring and the $\operatorname{Spin}(32) / \mathbf{Z}_{2}$ and $E_{8} \times E_{8}$ heterotic string theories. In the type II superstring, the massless bosonic states are the graviton multiplet, while they can also be gauge bosons in the heterotic theories. The corresponding amplitudes are functions of the 10-dimensional momenta $k_{i}=\left(k_{i}^{\mu}\right)$ and polarization tensors $\epsilon_{i}=\left(\epsilon_{i}^{\mu}\right)$ of the $N$ massless states, $0 \leq \mu \leq 9,1 \leq i \leq N$. Henceforth we restrict to genus 2 , so the moduli space $\mathcal{M}_{2}$ has dimension 3 .

2.1. General form of the scattering amplitudes. We concentrate on the amplitude of gravitons in the type II superstring, which we denote by $\mathbf{A}_{I I}\left(k_{i}, \epsilon_{i}\right)$, the others following by combining the holomorphic factors of $\mathbf{A}_{I I}\left(k_{i}, \epsilon_{i}\right)$ with the chiral correlators of gauge bosons, which can be computed directly. By the chiral splitting theorem of [28], the amplitude $\mathbf{A}_{I I}\left(k_{i}, \epsilon_{i}\right)$ is of the form

$$
\mathbf{A}_{I I}\left(k_{i}, \epsilon_{i}\right)=\int d p_{I}^{\mu} \int_{\mathcal{M}_{2} \times \Sigma^{N}} \mathcal{H}\left(z_{i} ; k_{i}, \epsilon_{i} ; p_{I}^{\mu}\right) \wedge \overline{\mathcal{H}\left(z_{i} ; k_{i}, \epsilon_{i} ; p_{I}^{\mu}\right)}
$$

Here we have fixed a canonical homology basis $A_{I}, B_{I}$, \# $\left(A_{I} \cap A_{J}\right)=$ $\#\left(B_{I} \cap B_{J}\right)=0$, \# $\left(A_{I} \cap B_{J}\right)=\delta_{I J}$. Let $\omega_{I}(z)$ be the basis of holomorphic $(1,0)$-forms dual to the $A_{I}$ cycles, and set $\Omega_{I J}=\oint_{B_{I}} \omega_{J}$. The moduli space $\mathcal{M}_{2}$ is identified with a fundamental domain of $\operatorname{Sp}(4, \mathbf{Z})$

${ }^{1}$ Since the version of this paper was submitted for publication in Current Developments in Mathematics in late September 2005, there have been several advances in the pure spinor formulation $[\mathbf{5 1}]$. 
in the Siegel domain of symmetric matrices $\Omega_{I J}$ with positive imaginary part. The parameters $p_{I}^{\mu}, 1 \leq I \leq h, 0 \leq \mu \leq 9$ are internal loop momenta. The expression $\mathcal{H}\left(z_{i} ; k_{i}, \epsilon_{i} ; p_{I}^{\mu}\right)$ is a $\Lambda^{3,0}\left(\mathcal{M}_{2}\right) \otimes\left(\otimes_{i=1}^{N} \Lambda_{z_{i}}^{1,0}(\Sigma)\right)$ form on $\mathcal{M}_{2} \times \Sigma^{N}$ which is holomorphic in both moduli and insertion points $z_{j}$ away from $z_{j} \neq z_{k}$, but which are twisted by the following monodromy as a point $z_{j}$ is transported along a closed cycle

$$
\begin{aligned}
\mathcal{H}\left(z_{i}+\delta_{i j} A_{K} ; k_{i}, \epsilon_{i} ; p_{I}^{\mu}\right) & =\mathcal{H}\left(z_{i} ; k_{i}, \epsilon_{i} ; p_{I}^{\mu}\right) \\
\mathcal{H}\left(z_{i}+\delta_{i j} B_{K} ; k_{i}, \epsilon_{i} ; p_{I}^{\mu}\right) & =\mathcal{H}\left(z_{i} ; k_{i}, \epsilon_{i} ; p_{I}^{\mu}+\delta_{I K} k_{j}^{\mu}\right) .
\end{aligned}
$$

The problem of evaluating the amplitude $\mathbf{A}_{I I}$ reduces to that of determining $\mathcal{H}\left(z_{i} ; k_{i}, \epsilon_{i} ; p_{I}^{\mu}\right)$. The holomorphicity of the desired form $\mathcal{H}\left(z_{i}\right.$; $\left.k_{i}, \epsilon_{i} ; p_{I}^{\mu}\right)$ is an essential requirement for the construction of heterotic string theories.

\subsection{Holomorphic $\mathcal{H}$, chiral $\mathcal{B}[\delta]$, and Dolbeault cohomology.} The following algorithm, based on a Dolbeault cohomology procedure, gives a solution to the problem of finding $\mathcal{H}\left(z_{i} ; k_{i}, \epsilon_{i} ; p_{I}^{\mu}\right)[\mathbf{9}]$.

For each even spin structure $\delta$ on $\Sigma$, there exists a form $\mathcal{B}[\delta]\left(z_{i} ; k_{i}, \epsilon_{i}\right.$; $\left.p_{I}^{\mu}\right)$ which is a correlation function on the worldsheet $\Sigma$ and which can itself be evaluated explicitly. We shall give the full prescription for $\mathcal{B}[\delta]\left(z_{i} ; k_{i}, \epsilon_{i} ; p_{I}^{\mu}\right)$ in the next section, but for the moment, we stress that $\mathcal{B}[\delta]\left(z_{i} ; k_{i}, \epsilon_{i} ; p_{I}\right)$ is a closed form in each $z_{i}$, and that for $N \geq 1$, $\mathcal{B}[\delta]\left(z_{i} ; k_{i}, \epsilon_{i} ; p_{I}^{\mu}\right)$ is a 1 -form in each point $z_{i}$ which may incorporate $(0,1)$-components. For such forms $\mathcal{B}[\delta]\left(z_{i} ; k_{i}, \epsilon_{i} ; p_{I}^{\mu}\right)$, there is no notion of holomorphicity. These forms arise from the chiral splitting theorem of $[\mathbf{2 8}]$ and are sometimes referred to as "chiral", since they are built only from correlations functions of chiral spinors on $\Sigma$. However, we stress that they are in general not holomorphic in $z_{i}$.

- Consider first the $N$-point function with $N=0$, which corresponds to the cosmological constant. Then there are no insertion points $z_{i}$, and $\mathcal{B}[\delta]\left(z_{i} ; k_{i}, \epsilon_{i} ; p_{I}^{\mu}\right)$ is a holomorphic function $\mathcal{B}[\delta]$ on $\mathcal{M}_{2}$. The relative phases $\epsilon_{\delta}{ }^{2}$ can be determined by the requirement that $\mathcal{H} \equiv \sum_{\delta} \epsilon_{\delta} \mathcal{B}[\delta]$ transforms so that the expression $\mathbf{A}_{I I}$ of (2.1) be modular invariant. The summation over spin structures $\delta$ is the Gliozzi-Scherk-Olive projection, and, physically, it is necessary to project out tachyonic states and insure space-time supersymmetry.

- Once the phases $\epsilon_{\delta}$ have been determined by the 0-point function, we can consider the sums $\sum_{\delta} \epsilon_{\delta} \mathcal{B}[\delta]\left(z_{i} ; k_{i}, \epsilon_{i} ; p_{I}^{\mu}\right)$ directly for $N \geq 1$. Then $\sum_{\delta} \epsilon_{\delta} \mathcal{B}[\delta]\left(z_{i} ; k_{i}, \epsilon_{i} ; p_{I}^{\mu}\right)=0$ for $N \leq 3$, while for $N=4$, there exist forms $\mathcal{S}_{j}\left(z_{i} ; k_{i}, \epsilon_{i} ; p_{I}^{\mu}\right)$ which are scalars in $z_{j}$ and closed 1-forms in $z_{i}$ for

${ }^{2}$ The phases $\epsilon_{\delta}$ should not be confused with the polarization tensors $\epsilon_{i}$ of the external states. Both notations are standard, which is why they have been kept. 
$i \neq j$ so that

$$
\sum_{\delta} \epsilon_{\delta} \mathcal{B}[\delta]\left(z_{i} ; k_{i}, \epsilon_{i} ; p_{I}^{\mu}\right)-\sum_{j=1}^{4} d \bar{z}_{j} \partial_{\bar{z}_{j}} \mathcal{S}_{j}\left(z_{i} ; k_{i}, \epsilon_{i} ; p_{I}^{\mu}\right) \in \bigotimes_{i=1}^{4} \Lambda_{z_{i}}^{1,0}(\Sigma) .
$$

The form $\mathcal{H}\left(z_{i} ; k_{i}, \epsilon_{i} ; p_{I}^{\mu}\right)$ can now be obtained by

$$
\mathcal{H}\left(z_{i} ; k_{i}, \epsilon_{i} ; p_{I}^{\mu}\right)=\sum_{\delta} \epsilon_{\delta} \mathcal{B}[\delta]\left(z_{i} ; k_{i}, \epsilon_{i} ; p_{I}^{\mu}\right)-\sum_{j=1}^{4} d_{j} \mathcal{S}_{j}\left(z_{i} ; k_{i}, \epsilon_{i} ; p_{I}^{\mu}\right),
$$

where $d_{j}$ is the de Rham exterior differential in each variable $z_{j}$. The closedness of $\mathcal{B}[\delta]\left(z_{i} ; k_{i}, \epsilon_{i} ; p_{I}^{\mu}\right)$ implies that $\mathcal{H}\left(z_{i} ; k_{i}, z_{i} ; p_{I}^{\mu}\right)$ is automatically holomorphic in each $z_{i}$.

We shall see below that the chiral forms $\mathcal{B}[\delta]\left(z_{i} ; k_{i}, \epsilon_{i} ; p_{I}^{\mu}\right)$ arise from superholomorphic forms with respect to a supergeometry $\left(g_{m n}, \chi_{m}{ }^{\alpha}\right)$. The above Dolbeault cohomology procedure solves an old puzzle: there is no relation between superholomorphicity and holomorphicity with respect to $g_{m n}$, but there is a deformed metric $\hat{g}_{m n}$ with respect to which holomorphic forms can be extracted from superholomorphic forms modulo forms which are Dolbeault-exact in one and de Rham-closed in the other insertion points.

2.3. The forms $\mathcal{B}[\delta]$ in terms of Green's functions. The amplitudes $\mathcal{B}[\delta]\left(z_{i} ; k_{i}, \epsilon_{i} ; p_{I}^{\mu}\right)$ are to be determined by taking the chiral contributions of functional integrals over all fluctuating worldsheets and all insertion points $z_{i}$ for the emission of the $N$ massless bosons, and factoring out correctly the gauge symmetries to arrive at well-defined, finite-dimensional integrals.

The basic result is that, by following the gauge-fixing procedure outlined in Section $\S 3$, the $\mathcal{B}[\delta]\left(z_{i} ; k_{i}, \epsilon_{i} ; p_{I}^{\mu}\right)$ are found to be $[\mathbf{9}]$

$$
\mathcal{B}[\delta]=\mathcal{B}[\delta]^{(d)}+\mathcal{B}[\delta]^{(c)} .
$$

Here the "connected" and "disconnected" components $\mathcal{B}[\delta]^{(d)}$ and $\mathcal{B}[\delta]^{(c)}$ are given in terms of two basic measures $d \mu_{2}[\delta]$ and $d \mu_{0}[\delta]$ on the moduli space $\mathcal{M}_{2}$ and Wick contractions of vertex operators $\mathcal{V}^{(0)}, \mathcal{V}^{(1)}$, and $\mathcal{V}^{(2)}$. The vertex operators $\mathcal{V}^{(0)}, \mathcal{V}^{(1)}, \mathcal{V}^{(2)}$ are defined by

$$
\begin{aligned}
& \mathcal{V}^{(0)}(z)=\epsilon^{\mu} d z\left(\partial_{z} x_{+}^{\mu}-i k^{\nu} \psi_{+}^{\mu} \psi_{+}^{\nu}\right)(z) \exp \left(i k \cdot x_{+}(z)\right) \\
& \mathcal{V}^{(1)}(z)=-\frac{1}{2} \epsilon^{\mu} d \bar{z} \chi_{\bar{z}}{ }^{+} \psi_{+}^{\mu}(z) \exp \left(i k \cdot x_{+}(z)\right) \\
& \mathcal{V}^{(2)}(z)=-\epsilon^{\mu} \hat{\mu}_{\bar{z}}^{z} d \bar{z}\left(\partial_{z} x_{+}^{\mu}-i k^{\nu} \psi_{+}^{\mu} \psi_{+}^{\nu}\right)(z) \exp \left(i k \cdot x_{+}(z)\right)
\end{aligned}
$$

where $x_{+}^{\mu}$ is an effective chiral scalar field with propagator $\left\langle x_{+}^{\mu}(z) x_{+}^{\nu}(w)\right\rangle$ $=-\delta^{\mu \nu} \ln E(z, w), E(z, w)$ being the prime form on the Riemann surface $\Sigma$. The spin structure $\delta$ determines a square root $\Lambda^{\frac{1}{2}, 0}[\delta](\Sigma)$ of the 
canonical bundle of $\Sigma$. The gravitino field $\chi(z)=\left(\chi_{m}{ }^{\alpha}\right)$ is a section of $\Lambda^{0,1}(\Sigma) \otimes \Lambda^{-\frac{1}{2}, 0}[\delta](\Sigma)$. It is given by $\chi(z)=\sum_{\alpha=1}^{2} \zeta^{\alpha} \chi_{\alpha}(z)$ where $\chi_{\alpha}(z)$ are two fixed, generic, but otherwise arbitrary sections of $\Lambda^{0,1}(\Sigma) \otimes$ $\Lambda^{-\frac{1}{2}, 0}[\delta](\Sigma), \zeta^{\alpha}$ are two anti-commuting parameters (corresponding to the odd supermoduli of $s \mathcal{M}_{2}$ to be discussed in Section $\left.\S 3\right)$, and $\hat{\mu}(z)=$ $\left(\hat{\mu}_{\bar{z}}{ }^{z}\right) \in \Lambda^{-1,1}(\Sigma)$ is a Beltrami differential defined modulo $\bar{\partial} T^{1,0}(\Sigma)$ by the condition

$$
\frac{1}{8 \pi} \int_{\Sigma} \int_{\Sigma} d^{2} z d^{2} w \omega_{I}(z) \chi(z) S_{\delta}(z, w) \chi(w) \omega_{J}(w)=\int_{\Sigma} \omega_{I}(z) \omega_{J}(z) \hat{\mu}(z),
$$

where $S_{\delta}(z, w)$ is the Szegö kernel. The measures $d \mu_{2}[\delta]$ and $d \mu_{0}[\delta]$ on $\mathcal{M}_{2}$ are defined by

$$
\begin{aligned}
& d \mu_{0}[\delta](\Omega)=\mathcal{Z}[\delta] \prod_{I \leq J} d \Omega_{I J} \\
& d \mu_{2}[\delta](\Omega)=\mathcal{Z}[\delta] \sum_{j=1}^{6} \mathcal{X}_{j} \prod_{I \leq J} d \Omega_{I J}
\end{aligned}
$$

with the following expressions for $\mathcal{Z}[\delta]$ and $\mathcal{X}_{j}, 1 \leq j \leq 6$ :

$$
\mathcal{Z}[\delta]=\frac{\left\langle\prod_{a} b\left(p_{a}\right) \prod_{\alpha} \delta\left(\beta\left(q_{\alpha}\right)\right)\right\rangle}{\operatorname{det}\left(\omega_{I} \omega_{J}\left(p_{a}\right)\right) \cdot\left\langle\chi_{\alpha} \mid \psi_{\beta}^{*}\right\rangle},
$$

where $p_{a}, q_{\alpha}$ are two sets of respectively 3 and 2 arbitrary generic points, and $\psi_{\beta}^{*}$ are the holomorphic forms of weight $3 / 2$ normalized at the points $q_{\alpha}$ by $\psi_{\beta}^{*}\left(q_{\alpha}\right)=\delta_{\alpha \beta}$. The fields $b(z)=b_{z z}, \beta(z)=\beta_{z+}$ and their partners $c(z)=c^{z}, \gamma(z)=\gamma^{+}$are the so-called superghost fields, with propagators

$$
\langle b(z) c(w)\rangle=G_{2}(z, w), \quad\langle\beta(z) \gamma(w)\rangle=-G_{3 / 2}(z, w)
$$

where $G_{n}(z, w)$ are the Green's functions on tensors of weight $n$. Next, let $S(z)=S_{z+}$ and $T(z)=T_{z z}$ be the supercurrent and the stress tensor defined by

$$
\begin{aligned}
& S(z)=-\frac{1}{2} \psi_{+}^{\mu} \partial_{z} x_{+}^{\mu}+\frac{1}{2} b \gamma-\frac{3}{2} \beta \partial_{z} c-\left(\partial_{z} \beta\right) c \\
& T(z)=-\frac{1}{2} \partial_{z} x^{\mu} \partial_{z} x^{\mu}+\frac{1}{2} \psi_{+}^{\mu} \partial_{z} \psi_{+}^{\mu}+c \partial_{z} b+2\left(\partial_{z} c\right) b-\frac{1}{2} \gamma \partial_{z} \beta-\frac{3}{2}\left(\partial_{z} \gamma\right) \beta .
\end{aligned}
$$


Then the expressions $\mathcal{X}_{j}, 1 \leq j \leq 6$, are given by

$$
\begin{aligned}
\mathcal{X}_{1} & =-\frac{1}{8 \pi^{2}} \int d^{2} z \chi_{\bar{z}}{ }^{+} \int d^{2} w \chi_{\bar{w}}{ }^{+}\langle S(z) S(w)\rangle \\
\mathcal{X}_{2}+\mathcal{X}_{3} & =+\frac{1}{16 \pi^{2}} \int d^{2} z \int d^{2} w \chi_{\bar{z}}{ }^{+} \chi_{\bar{w}}{ }^{+} T^{I J} \omega_{I}(z) S_{\delta}(z, w) \omega_{J}(w) \\
\mathcal{X}_{4} & =+\frac{1}{16 \pi^{2}} \int d^{2} w \partial_{p_{a}} \partial_{w} \ln E\left(p_{a}, w\right) \chi_{\bar{w}}{ }^{+} \int d^{2} u S_{\delta}(w, u) \chi_{\bar{u}}{ }^{+} \varpi_{a}^{*}(u) \\
\mathcal{X}_{5} & =+\frac{1}{16 \pi^{2}} \int d^{2} u \int d^{2} v S_{\delta}\left(p_{a}, u\right) \chi_{\bar{u}}{ }^{+} \partial_{p_{a}} S_{\delta}\left(p_{a}, v\right) \chi_{\bar{v}}{ }^{+} \varpi_{a}(u, v) \\
\mathcal{X}_{6} & =\frac{1}{16 \pi^{2}} \int d^{2} z \chi_{\alpha}^{*}(z) \int d^{2} w G_{3 / 2}(z, w) \chi_{\bar{w}}{ }^{+} \int d^{2} v \chi_{\bar{v}}{ }^{+} \Lambda_{\alpha}(w, v)
\end{aligned}
$$

where $\Lambda_{\alpha}(w, v)=2 G_{2}(w, v) \partial_{v} \psi_{\alpha}^{*}+3 \partial_{v} G_{2}(w, v) \psi_{\alpha}^{*}(v)$, the sections $\chi \beta^{*}(z)$ are the linear combinations of the sections $\chi_{\alpha}(z)$ normalized by $\left\langle\chi_{\beta}^{*} \mid \psi_{\alpha}^{*}\right\rangle$ $=\delta_{\alpha \beta}$, and $T^{I J}$ are the coefficients of the holomorphic quadratic differential defined by

$$
\begin{aligned}
& T^{I J} \omega_{I} \omega_{J}(w) \\
& =\frac{\left\langle T(w) \prod_{a=1}^{3} b\left(p_{a}\right) \prod_{\alpha=1}^{2} \delta\left(\beta\left(q_{\alpha}\right)\right)\right\rangle}{\left\langle\prod_{a=1}^{3} b\left(p_{a}\right) \prod_{\alpha=1}^{2} \delta\left(\beta\left(q_{\alpha}\right)\right)\right\rangle}-2 \sum_{a=1}^{3} \partial_{p_{a}} \partial_{w} \ln E\left(p_{a}, w\right) \varpi_{a}^{*}(w) \\
& \quad+\int d^{2} z \chi_{\alpha}^{*}(z)\left(-\frac{3}{2} \partial_{w} G_{3 / 2}(z, w) \psi_{\alpha}^{*}(w)-\frac{1}{2} G_{3 / 2}(z, w) \partial \psi_{\alpha}^{*}(w)\right. \\
& \left.\quad+G_{2}(w, z) \partial_{z} \psi_{\alpha}^{*}(z)+\frac{3}{2} \partial_{z} G_{2}(w, z) \psi_{\alpha}^{*}(z)\right),
\end{aligned}
$$

and $\varpi_{a}^{*}$ and $\varpi_{a}$ are holomorphic forms in $u$ and $v$ defined by $\varpi_{a}^{*}(u)=$ $\varpi_{a}\left(u, p_{a}\right)$ and

$$
\begin{aligned}
\varpi_{a}(u, v) & =\frac{\operatorname{det}\left\{\omega_{I} \omega_{J}\left(p_{b}[u, v ; a]\right)\right\}}{\operatorname{det}\left\{\omega_{I} \omega_{J}\left(p_{b}\right)\right\}} \\
\omega_{I} \omega_{J}\left(p_{b}[u, v ; a]\right) & = \begin{cases}\omega_{I} \omega_{J}\left(p_{b}\right) & \text { if } b \neq a \\
\frac{1}{2}\left(\omega_{I}(u) \omega_{J}(v)+\omega_{I}(v) \omega_{J}(u)\right) & \text { if } b=a\end{cases}
\end{aligned}
$$

In (2.13), all the apparent poles cancel, which is why $T^{I J}$ is well-defined. In the expressions for $\varpi_{a}^{*}$ and $\varpi_{a}$, the indices $I J$ and $a$ are both 3dimensional, and hence it makes sense to take the $3 \times 3$ determinants indicated. 
We can now give the expressions for $\mathcal{B}[\delta]^{(c)}$ and $\mathcal{B}[\delta]^{(1)}$,

$$
\begin{aligned}
\mathcal{B}[\delta]^{(d)} & =d \mu_{2}[\delta]\left\langle Q\left(p_{I}\right) \prod_{i=1}^{N} \mathcal{V}_{i}^{(0)}\left(z_{i} ; k_{i}\right)\right\rangle \\
\mathcal{B}[\delta]^{(c)} & =d \mu_{0}[\delta] \int \prod_{\alpha=1}^{2} d \zeta^{\alpha} \sum_{j=1}^{5} \mathcal{Y}_{j},
\end{aligned}
$$

where $Q\left(p_{I}\right)=\exp \left(i p_{I}^{\nu} \oint_{B_{I}} d z \partial_{z} x_{+}^{\nu}(z)\right)$, and

$$
\begin{aligned}
& \mathcal{Y}_{1}=\frac{1}{8 \pi^{2}}\left\langle Q\left(p_{I}\right) \int \chi S \int \chi S \prod_{j=1}^{N} \mathcal{V}_{j}^{(0)}\right\rangle_{(c)} \\
& \mathcal{Y}_{2}=\frac{1}{2 \pi}\left\langle Q\left(p_{I}\right) \int \hat{\mu} T \prod_{j=1}^{N} \mathcal{V}_{j}^{(0)}\right\rangle \\
& \mathcal{Y}_{3}=\frac{1}{2 \pi} \sum_{i=1}^{N}\left\langle Q\left(p_{I}\right) \int \chi S \mathcal{V}_{i}^{(1)} \prod_{j \neq i} \mathcal{V}_{j}^{(0)}\right\rangle \\
& \mathcal{Y}_{4}=\frac{1}{2}\left\langle Q\left(p_{I}\right) \mathcal{V}_{i}^{(1)} \mathcal{V}_{j}^{(1)} \prod_{l \neq i, j} \mathcal{V}_{l}^{(0)}\right\rangle \\
& \mathcal{Y}_{5}=\sum_{i=1}^{N}\left\langle Q\left(p_{I}\right) \mathcal{V}_{i}^{(2)} \prod_{j \neq i} \mathcal{V}_{j}^{(0)}\right\rangle .
\end{aligned}
$$

The preceding formulas give a complete and systematic way of obtaining the scattering amplitude for $N$ massless bosons to two-loop order. Their interpretation is roughly as follows. The choice $\chi(z)=$ $\sum_{\alpha=1}^{2} \zeta^{\alpha} \chi_{\alpha}(z)$ is a choice of gauge slice. The fundamental guiding principle of our gauge-fixing method is to project the supergeometry $\left(g_{m n}, \chi_{m}{ }^{\alpha}\right)$ on a super period matrix invariant under supersymmetry, rather than on the metric $g_{m n}$. Since the functional integrals are originally defined in terms of the metric $g_{m n}$, this requires a deformation of complex structures implemented through the Beltrami differential $\hat{\mu}(z)$. The terms $\mathcal{X}_{j}, 2 \leq j \leq 5$, incorporate both local and global effects of this deformation of complex structures. In general, the emission of a string state is implemented by insertion of a vertex, in this case, the vertex $\mathcal{V}^{(0)}$ which is the naive vertex for graviton emission. However, due to the gauge-fixing procedure and the deformation of complex structures, the naive vertex must be corrected by the vertices $\mathcal{V}^{(1)}$ and $\mathcal{V}^{(2)}$. This produces the terms $\mathcal{Y}_{j}, 2 \leq j \leq 5$. Note that $\mathcal{V}^{(0)}$ is a $(1,0)$-form, but $\mathcal{V}^{(1)}, \mathcal{V}^{(2)}$ are $(0,1)$-forms. The period matrix $\Omega_{I J}$ of the previous formulas is actually the period matrix $\hat{\Omega}_{I J}$ of the metric $\hat{g}_{m n}$, but after the deformation of complex structures, we drop the "hat" notation for simplicity. 
2.4. Gauge--slice independence of the measure $d \mu_{2}[\delta](\Omega)$. Next, the amplitudes $\mathbf{A}_{I I}$ have to be shown to be independent of all the choices of $q_{\alpha}, p_{a}, \chi_{\alpha}(z), \mu(z)$ entering the amplitudes $\mathcal{B}[\delta]\left(z_{i} ; k_{i}, \epsilon_{i} ; k_{I}^{\mu}\right)$. This is important because it had not been satisfied by earlier Ansätze, and there had been concern that superstring scattering amplitudes could be ambiguous. It also paves the way for the evaluation of $\mathcal{B}[\delta]\left(z_{i} ; k_{i}, \epsilon_{i}\right.$; $\left.p_{I}^{\mu}\right)$ in terms of $\vartheta$-functions.

We begin with the measure $d \mu_{2}[\delta](\Omega)[6,7]$. The gauge slice independence of $d \mu_{2}[\delta](\Omega)$ is established by showing that its variational derivative with respect to any of the above choices vanishes identically on the moduli space $\mathcal{M}_{2}$ [6]. The following special case is of considerable practical value, and produces relatively simpler expressions which can independently be shown to be independent of all remaining choices. Choose $\chi_{\alpha}(z)$ to be a Dirac measure at a point $x_{\alpha}$ and let $x_{\alpha} \rightarrow q_{\alpha}$. All dependence on $\mu(z)$ cancels out completely, and the resulting expression for $d \mu_{2}[\delta]$ becomes

$$
d \mu_{2}[\delta]=\mathcal{Z}[\delta] \sum_{j=1}^{6} \mathcal{X}_{j}
$$

with

$$
\mathcal{Z}[\delta]=\frac{\left\langle\prod_{a=1}^{3} b\left(p_{a}\right) \prod_{\alpha=1}^{2} \delta\left(\beta\left(q_{\alpha}\right)\right)\right\rangle}{\operatorname{det} \omega_{I} \omega_{J}\left(p_{a}\right)}
$$

and the terms $\mathcal{X}_{i}$ given by

$$
\begin{aligned}
\mathcal{X}_{1}+\mathcal{X}_{6}= & \frac{\zeta^{1} \zeta^{2}}{16 \pi^{2}}\left[-10 S_{\delta}\left(q_{1}, q_{2}\right) \partial_{q_{1}} \partial_{q_{2}} \ln E\left(q_{1}, q_{2}\right)\right. \\
& -\partial_{q_{1}} G_{2}\left(q_{1}, q_{2}\right) \partial \psi_{1}^{*}\left(q_{2}\right)+\partial_{q_{2}} G_{2}\left(q_{2}, q_{1}\right) \partial \psi_{2}^{*}\left(q_{1}\right) \\
& \left.+2 G_{2}\left(q_{1}, q_{2}\right) \partial \psi_{1}^{*}\left(q_{2}\right) f_{3 / 2}^{(1)}\left(q_{2}\right)-2 G_{2}\left(q_{2}, q_{1}\right) \partial \psi_{2}^{*}\left(q_{1}\right) f_{3 / 2}^{(2)}\left(q_{1}\right)\right] \\
\mathcal{X}_{2}= & \frac{\zeta^{1} \zeta^{2}}{16 \pi^{2}} \omega_{I}\left(q_{1}\right) \omega_{J}\left(q_{2}\right) S_{\delta}\left(q_{1}, q_{2}\right)\left[\partial_{I} \partial_{J} \ln \frac{\vartheta[\delta](0)^{5}}{\vartheta[\delta]\left(D_{\beta}\right)}+\partial_{I} \partial_{J} \ln \vartheta\left(D_{b}\right)\right] \\
\mathcal{X}_{3}= & \frac{\zeta^{1} \zeta^{2}}{8 \pi^{2}} S_{\delta}\left(q_{1}, q_{2}\right) \sum_{a} \varpi_{a}\left(q_{1}, q_{2}\right)\left[B_{2}\left(p_{a}\right)+B_{3 / 2}\left(p_{a}\right)\right] \\
\mathcal{X}_{4}= & \frac{\zeta^{1} \zeta^{2}}{8 \pi^{2}} S_{\delta}\left(q_{1}, q_{2}\right) \sum_{a}\left[\partial_{p_{a}} \partial_{q_{1}} \ln E\left(p_{a}, q_{1}\right) \varpi_{a}^{*}\left(q_{2}\right)\right. \\
& \left.+\partial_{p_{a}} \partial_{q_{2}} \ln E\left(p_{a}, q_{2}\right) \varpi_{a}^{*}\left(q_{1}\right)\right] \\
\mathcal{X}_{5}= & \frac{\zeta^{1} \zeta^{2}}{16 \pi^{2}} \sum_{a}\left[S_{\delta}\left(p_{a}, q_{1}\right) \partial_{p_{a}} S_{\delta}\left(p_{a}, q_{2}\right)\right. \\
& \left.-S_{\delta}\left(p_{a}, q_{2}\right) \partial_{p_{a}} S_{\delta}\left(p_{a}, q_{1}\right)\right] \varpi_{a}\left(q_{1}, q_{2}\right) .
\end{aligned}
$$


Here $D_{b}=p_{1}+p_{2}+p_{3}-3 \Delta, D_{\beta}=q_{1}+q_{2}-2 \Delta$, and the expressions $f_{n}(w), f_{3 / 2}^{(1)}(x), f_{3 / 2}^{(2)}(x), B_{2}(w)$ and $B_{3 / 2}(w)$ are given by

$$
\begin{aligned}
f_{n}(w)= & \omega_{I}(w) \partial_{I} \ln \vartheta[\delta]\left(D_{n}\right)+\partial_{w} \ln \left(\sigma(w)^{2 n-1} \prod_{i=1}^{2 n-1} E\left(w, z_{i}\right)\right) \\
f_{3 / 2}^{(1)}(x)= & \omega_{I}\left(q_{1}\right) \partial_{I} \ln \vartheta[\delta]\left(x+q_{2}-2 \Delta\right) \\
& +\partial_{q_{1}} \ln \left(E\left(q_{1}, q_{2}\right) E\left(q_{1}, x\right) \sigma\left(q_{1}\right)^{2}\right) \\
f_{3 / 2}^{(2)}(x)= & \omega_{I}\left(q_{2}\right) \partial_{I} \ln \vartheta[\delta]\left(x+q_{1}-2 \Delta\right) \\
& +\partial_{q_{2}} \ln \left(E\left(q_{2}, q_{1}\right) E\left(q_{2}, x\right) \sigma\left(q_{2}\right)^{2}\right) \\
B_{2}(w)= & -2 T_{1}(w)+\frac{1}{2} f_{2}(w)^{2}-\frac{3}{2} \partial_{w} f_{2}(w) \\
& -2 \sum_{a} \partial_{p_{a}} \partial_{w} \ln E\left(p_{a}, w\right) \varpi_{a}^{*}(w)
\end{aligned}
$$

$(2.20) \quad B_{3 / 2}(w)=12 T_{1}(w)-\frac{1}{2} f_{3 / 2}(w)^{2}+\partial f_{3 / 2}(w)$

with $\Delta$ the vector of Riemann constants, $\sigma(z)$ the basic function with monodromy introduced in $[\mathbf{2 9}, \mathbf{3 0}, \mathbf{3 1}]$, and $E(z, w)=(z-w)+(z-$ $w)^{2} T_{1}(w)+O\left((z-w)^{3}\right)$ defining the chiral scalar bosonic stress tensor $-T_{1}(w)$.

Compared with the earlier expression $(2.12)$ for $\mathcal{X}_{j}$ and for $d \mu_{2}[\delta]$, all field theoretic correlation functions have been worked out, and the new expression only involves complex function theory on the Riemann surface $\Sigma$. It can be checked directly to be independent of the choice points $p_{a}, q_{\alpha}[\mathbf{7}]$.

The measure $d \mu_{2}[\delta]$ suffices to determine the $N=0$ amplitude, which is also the space-time cosmological constant. In fact, in this case, there is no vertex operator, and the internal momenta $p_{I}^{\mu}$ can be integrated out to give

$$
\left.\mathbf{A}_{I I}\right|_{N=0}=\int_{\mathcal{M}_{2}}(\operatorname{det} \operatorname{Im} \Omega)^{-5} \sum_{\delta} \epsilon_{\delta} d \mu_{2}[\delta](\Omega) \wedge \overline{\sum_{\delta} \epsilon_{\delta} d \mu_{2}[\delta](\Omega)}
$$

with the phases $\epsilon_{\delta}$ yet to be determined by modular invariance.

2.5. Gauge-slice independence of the $N$-point function. We consider next the slice-independence of the $N$-point function [9]. Since the correlator $\left\langle Q\left(p_{I}\right) \prod_{j=1}^{N} \mathcal{V}^{(0)}\left(z_{j}\right)\right\rangle$ is manifestly independent of any choice of gauge-slice, and since $d \mu_{2}[\delta]$ has been shown to be slice-independent, the term $\mathcal{B}[\delta]^{(d)}$ is slice-independent. 
The term $\mathcal{B}[\delta]^{(c)}$ is not invariant under changes of gauge slices, but it transforms by $[\mathbf{9}]$

$$
\mathcal{B}[\delta]^{(c)}\left(z_{i} ; k_{i}, \epsilon_{i} ; p_{I}\right) \rightarrow \mathcal{B}[\delta]^{(c)}\left(z_{i} ; k_{i}, \epsilon_{i} ; p_{I}\right)+\sum_{i=1}^{N} d_{i} \mathcal{R}_{i}[\delta]\left(z_{i} ; k_{i}, \epsilon_{i} ; p_{I}\right),
$$

where the forms $\mathcal{R}_{i}[\delta]\left(z_{i} ; k_{i}, \epsilon_{i} ; p_{I}^{\mu}\right)$ are scalars in $z_{i}$, de Rham closed forms in $z_{j}$ for $j \neq i$, and have the same monodromy as $\mathcal{B}[\delta]$. Since the forms $\mathcal{B}[\delta]$ are closed in each $z_{i}$, and since by analytic continuation $[\mathbf{3 2}]$, the singularities at coincident insertion points $z_{i}=z_{j}$ are harmless, it follows from a Riemann bilinear relations argument that the terms $\mathcal{R}_{i}[\delta]$ do not contribute to the integrated amplitudes $\mathbf{A}_{I I}\left(k_{i}, \epsilon_{i}\right)$. Thus the $N$-point functions $\mathbf{A}_{I I}\left(k_{i}, \epsilon_{i}\right)$ are gauge slice-independent.

2.6. The measure $d \mu_{2}[\delta]$ and the modular covariant form $\Xi_{6}[\delta]$. Once the gauge slice independence has been established, the chiral amplitudes $\mathcal{B}[\delta]$ can be evaluated explicitly by making convenient choices for the points $p_{a}, q_{\alpha}$.

The first fundamental term is $d \mu_{2}[\delta](\Omega)$, which is the chiral string measure, and which will determine the phases $\epsilon_{\delta}$. We find $[\mathbf{5}, \mathbf{8}]$

$$
d \mu_{2}[\delta](\Omega)=\frac{1}{16 \pi^{6}} \frac{\Xi_{6}[\delta](\Omega) \vartheta[\delta](\Omega)^{4}}{\Psi_{10}(\Omega)} \prod_{I \leq J} d \Omega_{I J}
$$

The form $\Psi_{10}(\Omega)$ is the familiar modular form of weight 10 defined by

$$
\Psi_{10}(\Omega)=\prod_{\delta \text { even }} \vartheta[\delta](\Omega)^{2} .
$$

The key new form is $\Xi_{6}[\delta](\Omega)$, whose construction depends on some particular properties of even spin structures in genus $h=2$. Recall that, in genus $h=2$, there are 10 even spin structures $\delta$ and 6 odd spin structures $\nu$, denoted by $\nu_{1}, \cdots, \nu_{6}$. Any even spin structure $\delta$ can be decomposed as a sum of 3 odd spin structures. If we write $\delta$ accordingly as $\delta=\nu_{1}+\nu_{2}+\nu_{3}$, then $\Xi_{6}[\delta](\Omega)$ is given by

$$
\Xi_{6}[\delta]=\sum_{1 \leq i<j \leq 3}\left\langle\nu_{i} \mid \nu_{j}\right\rangle \prod_{k=4,5,6} \vartheta\left[\nu_{i}+\nu_{j}+\nu_{k}\right]^{4}(\Omega) .
$$

A very important property of $\Xi_{6}[\delta](\Omega)$ is its transformation law under $S p(4, \mathbf{Z})$, which is not quite that a modular form, but rather

$$
\Xi_{6}[\tilde{\delta}](\tilde{\Omega})=\epsilon^{4} \operatorname{det}(C \Omega+D)^{2} \Xi_{6}[\delta](\Omega), \quad\left(\begin{array}{ll}
A & B \\
C & D
\end{array}\right) \in S p(4, \mathbf{Z}),
$$

where $\tilde{\Omega}=(A \Omega+B)(C \Omega+D)^{-1}, \tilde{\delta}$ is the corresponding transform of the spin structure $\delta$, and $\epsilon$ is exactly the same 8th-root of unity which occurs in the transformation law for $\vartheta$-constants, $\vartheta[\tilde{\delta}](\tilde{\Omega})=\epsilon^{4} \operatorname{det}(C \Omega+$ 
$D)^{6} \vartheta[\delta](\Omega)$. There would have been no such factors $\epsilon^{4}$ in the transformation law for modular forms. This shows that there is a unique choice of relative phases $\epsilon_{\delta}=+1$ between the various even spin structures for the GSO projection, given by $\sum_{\delta} d \mu[\delta](\Omega)$. By examining degenerations of the surface $\Sigma$, it is then not difficult to show that

$$
\sum_{\delta} \Xi_{6}[\delta](\Omega) \vartheta[\delta](\Omega)^{4}=0
$$

and hence $\sum_{\delta} d \mu_{2}[\delta](\Omega)=0$. Physically, this means that the cosmological constant vanishes in superstring theory, which is a consequence of space-time supersymmetry. Mathematically, for genus $h=1$, the vanishing of the cosmological constant was known to follow from the Jacobi identity for $\vartheta$-constants, and thus from the Riemann identities. In genus 2, however, the identity (2.27) does not follow from the Riemann identities alone. Rather, it is equivalent to the fact that an $\operatorname{Sp}(4, \mathbf{Z})$ modular form of weight 8 must be proportional to the square of the unique $S p(4, \mathbf{Z})$ modular form of weight 4 .

2.7. Explicit formula for the holomorphic form $\mathcal{H}$. Once the relative phases $\epsilon_{\delta}=1$ have been determined, we can evaluate directly the Gliozzi-Scherk-Olive sum $\sum_{\delta} \epsilon_{\delta} \mathcal{B}[\delta]=\sum_{\delta} \mathcal{B}[\delta]$ instead of evaluating each $\mathcal{B}[\delta]$ separately. Using now the unitary gauge with $q_{\alpha}$ the divisor of a holomorphic one form $\varpi(z)$, we find [10]

$$
\sum_{\delta} \mathcal{B}[\delta]\left(z_{i} ; k_{i}, \epsilon_{i} ; p_{I}^{\mu}\right)=0, \quad 0 \leq N \leq 3,
$$

while for $N=4$, we find

$$
\begin{aligned}
\sum_{\delta} \mathcal{B}[\delta]\left(z_{i} ; k_{i}, \epsilon_{i} ; p_{I}^{\mu}\right) & =\mathcal{H}\left(z_{i} ; k_{i}, \epsilon_{i} ; p_{I}^{\mu}\right) \\
& +\sum d_{i}\left(\Lambda\left(z_{i}\right)\left\langle Q\left(p_{I}\right) \prod_{j=1}^{4} e^{i k_{j} x_{+}\left(z_{j}\right)}\right\rangle \prod_{j \neq i} \varpi\left(z_{j}\right)\right)
\end{aligned}
$$

where $\Lambda(z)$ is a certain single-valued smooth scalar function, and the holomorphic form $\mathcal{H}\left(z_{i} ; k_{i}, \epsilon_{i} ; p_{I}^{\mu}\right)$ is given by

$$
\begin{aligned}
\mathcal{H}\left(z_{i} ; k_{i}, \epsilon_{i} ; p_{I}^{\mu}\right)=\frac{1}{64 \pi^{2}} & K \mathcal{Y}_{S} \exp \left(i \pi p_{I}^{\mu} \Omega_{I J} p_{J}^{\mu}\right. \\
& \left.+2 \pi i \sum_{j=1}^{4} p_{I}^{\mu} k_{j}^{\mu} \int^{z_{j}} \omega_{I}\right) \prod_{i<j} E\left(z_{i}, z_{j}\right)^{k_{i} \cdot k_{j}}
\end{aligned}
$$

where the factor $\mathcal{Y}_{S}$ is defined to be

$$
\begin{aligned}
3 \mathcal{Y}_{S}= & +\left(k_{1}-k_{2}\right) \cdot\left(k_{3}-k_{4}\right) \Delta\left(z_{1}, z_{2}\right) \Delta\left(z_{3}, z_{4}\right) \\
& +\left(k_{1}-k_{3}\right) \cdot\left(k_{2}-k_{4}\right) \Delta\left(z_{1}, z_{3}\right) \Delta\left(z_{2}, z_{4}\right) \\
& +\left(k_{1}-k_{4}\right) \cdot\left(k_{2}-k_{3}\right) \Delta\left(z_{1}, z_{4}\right) \Delta\left(z_{2}, z_{3}\right)
\end{aligned}
$$


and $\Delta(z, w)=\omega_{1}(z) \omega_{2}(w)-\omega_{1}(w) \omega_{2}(z)$ is the basic anti-symmetric biholomorphic form. The kinematic factor $K=K(1,2,3,4)$ is the same one as in tree-level and one-loop amplitudes. Explicitly, in terms of the gauge-invariant field strengths $f_{i}^{\mu \nu}=\epsilon_{i}^{\mu} k_{i}^{\nu}-\epsilon_{i}^{\nu} k_{i}^{\mu}$, it can be written as

$$
\begin{aligned}
K(1,2,3,4)= & \left(f_{1} f_{2}\right)\left(f_{3} f_{4}\right)+\left(f_{1} f_{3}\right)\left(f_{2} f_{4}\right)+\left(f_{1} f_{4}\right)\left(f_{2} f_{3}\right) \\
& -4\left(f_{1} f_{2} f_{3} f_{4}\right)-4\left(f_{1} f_{3} f_{2} f_{4}\right)-4\left(f_{1} f_{2} f_{4} f_{3}\right),
\end{aligned}
$$

with $\left(f_{i} f_{j}\right)=f_{i}^{\mu \nu} f_{j}^{\nu \mu},\left(f_{i} f_{j} f_{k} f_{l}\right)=f_{i}^{\mu \nu} f_{j}^{\nu \rho} f_{k}^{\rho \sigma} f_{l}^{\sigma \mu}$.

2.8. The 4-point function. Using equation (2.1), the 4-point function $\mathbf{A}_{I I}$ follows readily from the exact formula for $\mathcal{H}\left(z_{i} ; k_{i}, \epsilon_{i} ; p_{I}^{\mu}\right)$ which we just obtained. The integral over the internal momenta $p_{I}^{\mu}$ completes the factors $E\left(z_{i}, z_{j}\right)$ into Green's functions, and we obtain [10]

$$
\begin{aligned}
& \mathbf{A}_{I I}\left(k_{i}, \epsilon_{i}\right) \\
& =\frac{K \bar{K}}{2^{12} \pi^{4}} \int_{\mathcal{M}_{2} \times \Sigma^{4}} \frac{\left|\prod_{I \leq J} d \Omega_{I J}\right|^{2}}{(\operatorname{det} \operatorname{Im} \Omega)^{5}}\left|\mathcal{Y}_{S}\right|^{2} \exp \left(-\sum_{i<j} k_{i} \cdot k_{j} G\left(z_{i}, z_{j}\right)\right),
\end{aligned}
$$

where $G(z, w)$ is the conformally invariant Green's function

$$
G(z, w)=-\ln |E(z, w)|^{2}+2 \pi(\operatorname{Im} \Omega)_{I J}^{-1}\left(\operatorname{Im} \int_{z}^{w} \omega_{I}\right)\left(\operatorname{Im} \int_{z}^{w} \omega_{J}\right)
$$

An expression in the hyperelliptic representation equivalent to (2.33) was partly guessed in $[\mathbf{3 3}]$, starting also from the measures $d \mu_{2}[\delta]$ and $d \mu_{0}[\delta]$ given in $[\mathbf{5}, \mathbf{6}, \mathbf{7}, \mathbf{8}]$. The derivation in $[\mathbf{3 3}]$ is not gauge slice independent, however, because the corrections $\mathcal{V}^{(1)}$ and $\mathcal{V}^{(2)}$ to the vertex operators were not taken into account.

The 4-point functions for the heterotic string are obtained by replacing in (2.1), at common loop momenta $p_{I}^{\mu}$, the holomorphic factors by the holomorphic blocks of the 10-dimensional bosonic string coupled with 32 worldsheet chiral fermions. They are of the form

$$
\begin{aligned}
& \mathbf{A}_{H E T} \\
& =\frac{K \bar{K}}{2^{12} \pi^{4}} \int_{\mathcal{M}_{2} \times \Sigma^{4}} \frac{\left|\prod_{I \leq J} d \Omega_{I J}\right|^{2}}{\pi^{12} \Psi_{10}(\Omega)(\operatorname{det} \operatorname{Im} \Omega)^{5}} \mathcal{W}\left(z_{1}, z_{2}, z_{3}, z_{4}\right) \overline{\mathcal{Y}_{S}\left(z_{1}, z_{2}, z_{3}, z_{4}\right)} \\
& \quad \times \exp \left(-\sum_{i<j} k_{i} \cdot k_{j} G\left(z_{i}, z_{j}\right)\right),
\end{aligned}
$$

where the holomorphic block $\mathcal{W}\left(z_{1}, z_{2}, z_{3}, z_{4}\right)$ depends on the external states and can be written down explicitly. For example, for the relatively more complicated scattering of two gravitons and two gauge bosons, we 
have respectively $\mathcal{W}=\mathcal{W}_{\left(R^{2} F^{2}\right)}$ and $\mathcal{W}=\mathcal{W}_{\left(R^{4}\right)}$, with

$$
\begin{aligned}
\mathcal{W}_{\left(R^{2} F^{2}\right)}= & \mathcal{W}_{\left(F^{2}\right)}\left(z_{1}, z_{2}\right)\left\{\epsilon_{1}^{\mu} \epsilon_{2}^{\mu} \partial_{z_{3}} \partial_{z_{4}} G\left(z_{3}, z_{4}\right)\right. \\
& \left.-\sum_{i j} \epsilon_{3}^{\mu} k_{i}^{\mu} \epsilon_{4}^{\nu} k_{j}^{\nu} \partial_{z_{3}} G\left(z_{3}, z_{i}\right) \partial_{z_{4}} G\left(z_{4}, z_{j}\right)\right\} \\
\mathcal{W}_{\left(R^{4}\right)}= & \frac{\left\langle\prod_{j=1}^{4} \epsilon_{j}^{\mu} \partial x^{\mu}\left(z_{j}\right) e^{i k_{j} \cdot x\left(z_{j}\right)}\right\rangle}{\left\langle\prod_{j=1}^{4} e^{i k_{j} \cdot x\left(z_{j}\right)}\right\rangle}
\end{aligned}
$$

where $x(z, \bar{z})$ is a non-chiral scalar field with propagator $G(z, w)$, and

$$
\begin{aligned}
& \mathcal{W}_{\left(F^{2}\right)}\left(z_{1}, z_{2}\right)=\frac{1}{2} \operatorname{tr}\left(T^{a_{1}} T^{a_{2}}\right) \sum_{\kappa} \vartheta[\kappa]^{8} S_{\kappa}\left(z_{1}, z_{2}\right)^{2} \\
& \mathcal{W}_{\left(F^{2}\right)}\left(z_{1}, z_{2}\right)=\frac{1}{2} \operatorname{tr}\left(T^{a_{1}} T^{a_{2}}\right) \sum_{\kappa} \vartheta[\kappa]^{8} \sum_{\rho} \vartheta[\rho]^{4} S_{\rho}\left(z_{1}, z_{2}\right)^{2},
\end{aligned}
$$

depending on whether the heterotic theory is the $\operatorname{Spin}(32) / \mathbf{Z}_{2}$ or the $E_{8} \times E_{8}$ theory.

2.9. Non-renormalization theorems. The low-energy effective action of superstring theories provides corrections to the Einstein action involving higher order curvature terms as well as couplings to additional fields such as gauge bosons [34]. The amplitudes $\mathbf{A}_{I I}, \mathbf{A}_{H E T}$ we just obtained allow us to determine readily the two-loop corrections to terms such as $\mathcal{R}^{4}$ in the type II superstring, and $F^{4}, F^{2} F^{2}, \mathcal{R}^{2} F^{2}, \mathcal{R}^{4}$ in the heterotic strings [10]. Here $\mathcal{R}^{4}=t_{8} t_{8} R^{4}, R$ is the space-time Riemann curvature tensor, and $F$ is the curvature of the gauge bosons. In determining the low-energy corrections, we have to let $k_{i} \rightarrow 0$, but only after the amplitude has been expressed in terms of the field strengths $f_{i}^{\mu \nu}=\epsilon_{i}^{\mu} k_{i}^{\nu}-\epsilon_{i}^{\nu} k_{i}^{\mu}$. A strong motivation for determining these corrections are the conjectured dualities between the $\operatorname{Spin}(32) / \mathbf{Z}_{2}$ heterotic theory and the type I superstring, as well as the S-duality of the type IIB superstring (see the next section).

For the type II superstring, it is manifest from the explicit form of $\mathbf{A}_{I I}\left(k_{i}, \epsilon_{i}\right)$ that the two-loop contribution to $\mathcal{R}^{4}$ vanishes. The heterotic strings are more subtle, because the contributions of the bosonic left sector necessarily have poles in the Mandelstam variables $s_{i j}=-2 k_{i} \cdot k_{j}$. Nevertheless, we find that terms such as $s_{i j} \mathcal{W}_{\left(\mathcal{R}^{2}\right)}$ and $s_{i j} s_{l m} \mathcal{W}_{\left(\mathcal{R}^{4}\right)}$ can be expressed in expressions such as

$$
\begin{aligned}
\partial_{z_{1}} \partial_{z_{2}} G\left(z_{1}, z_{2}\right) \exp \left(-\sum_{i<j} k_{i} \cdot k_{j} G\left(z_{i}, z_{j}\right)\right) & \\
& \sum_{i<j} C_{i j}^{\mu \nu} \partial_{z_{1}} G\left(z_{1}, z_{i}\right) \partial_{z_{2}} G\left(z_{2}, z_{j}\right),
\end{aligned}
$$


whose integrals against holomorphic differentials tend to 0 as $k_{i} \cdot k_{j} \rightarrow 0$. This turns out to suffice to establish the desired non-renormalization theorem, by which the terms $\mathcal{R}^{2} F^{2}$ and $\mathcal{R}^{4}$ in the heterotic string do not receive corrections to two-loop order $[\mathbf{1 0}]$.

2.10. S-duality for the type IIB superstring. Here we discuss joint work with M. Gutperle on a partial check of the famous $S L(2, \mathbf{Z})$ dualities for the type IIB superstring conjectured by M. Green, M. Gutperle, P. Vanhove, H.G. Kwon, and others (see $[\mathbf{3 5}, \mathbf{3 6}, \mathbf{3 7}]$, and references in [14]). $S$-duality provides powerful constraints on the form of the low-energy effective actions. In particular, it was conjectured in [36] that the $D^{4} \mathcal{R}^{4}$ terms in the type IIB effective action are of the form

$$
S_{D^{4} R^{4}}=C_{D^{4} \mathcal{R}^{4}} \int d^{10} x \sqrt{-G} D^{4} \mathcal{R}^{4} e^{\frac{1}{2} \phi} 2 \zeta(5) E_{5 / 2}(\tau, \bar{\tau})
$$

where $\tau=\chi+i e^{-\phi}$ is the axion/dilaton field, $\zeta(s)$ is the Riemann zeta function, and $E_{5 / 2}(\tau, \bar{\tau})$ is the non-holomorphic Eisenstein series of weight $s=5 / 2$,

$$
2 \zeta(s) E_{s}(\tau, \bar{\tau})=\sum_{(m, n) \neq(0,0)} \frac{\tau_{2}^{s}}{|m+n \tau|^{2 s}} .
$$

Expanding $2 \zeta(5) E_{5 / 2}(\tau, \bar{\tau})$ in $\tau$, this conjecture predicts in particular the precise value of the contribution to the $D^{4} \mathcal{R}^{4}$ of the two-loop perturbative amplitude.

This prediction can be compared with that of the formula (2.33), which gives the two-loop amplitude up to an overall constant due to bosonization formulas. The precise value of this constant can be determined using factorization. We find that it matches exactly that predicted from Eisenstein series, and thus the perturbative two-loop amplitude provides a partial confirmation of the conjectured S-duality $[14]$.

2.11. Orbifolds and Kachru-Kumar-Silverstein models. So far, we have considered only superstrings evolving in flat Minkowski space-time. However, the preceding gauge-fixing procedure adapts readily to other space-times, simply by replacing the correlation functions of the fields $x_{+}^{\mu}, \psi_{+}^{\mu}$ by those of the corresponding conformal field theory [5]. Here we discuss joint work with K. Aoki on the cosmological constant of some orbifold models proposed by S. Kachru, S. Kumar, and E. Silverstein [15]. These KKS models are of particular interest since their supersymmetry is broken, yet their cosmological constant vanishes to one-loop. There was initially some hope that the cosmological constant would still vanish to two loops, but we can now show, using the new gauge-fixing method, that this is not the case [16]. 
The KKS models are constructed with an orbifold group $G$ generated by two elements $f=\left(\left(r_{L}, s_{R}\right)^{1-4},\left(1, s_{R}^{2}\right)^{5},\left(s_{L}, s_{R}\right)^{6} ;(-)^{F_{R}}\right), g=$ $\left.\left(s_{L}, s_{R}\right)^{1-4},\left(s_{L}, s_{R}\right)^{5},\left(s_{L}^{2}, 1\right)^{6} ;(-)^{F_{L}}\right)$ acting on a square torus with selfdual radius. Here $s_{L}, s_{R}, r_{L}, r_{R}$ are chiral and reflections acting on the left and right sectors, and the superscripts denote the dimension on which the operator acts. The orbifold action creates sectors for the theory, indexed by two twists $\epsilon, \alpha$, and the chiral string measure $d \mu_{2}[\delta]$ is replaced now in each $(\epsilon, \alpha)$ sector by the following measure,

$$
d \mu_{C}[\delta ; \epsilon, \alpha]\left(p_{L}\right)=\frac{e^{i \pi \tau_{\epsilon} p_{L}^{2}}}{16 \pi^{6} \Psi_{10}} \frac{\vartheta\left[\delta_{j}^{+}\right]^{2} \vartheta\left[\delta_{j}^{-}\right]^{2}}{\vartheta_{j}^{4}\left(0, \tau_{\epsilon}\right)} \sum_{\delta}\langle\alpha \mid \delta\rangle \Xi_{6}[\delta] \vartheta[\delta]^{2} \vartheta[\delta+\epsilon]^{2} .
$$

Here $\tau_{\epsilon}$ is the Prym period matrix associated to the twist $\epsilon$. In genus $h=$ 2 , the even spin structures $\delta$ fall into two groups, depending on whether $\delta+\epsilon$ is even or odd. The group with $\delta+\epsilon$ even consists of 6 elements, which can be divided themselves into $\delta_{i}^{+}$and $\delta_{j}^{+}, j=2,3,4, \delta_{j}^{-}=$ $\delta_{j}^{+}+\epsilon$. These are the spin structures occurring in the above formula for $d \mu_{C}[\delta ; \epsilon, \alpha]\left(p_{L}\right)$. The Schottky relations imply that the choice of $j$ is immaterial.

The asymptotic behavior of the measure $d \mu[\delta ; \epsilon, \alpha]\left(p_{L}\right)$ is now easily determined along the divisor of separating nodes. For example, in the

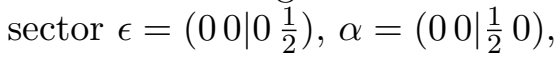

$$
\sum_{\delta}\langle\alpha \mid \delta\rangle \Xi_{6}[\delta] \vartheta[\delta]^{2} \vartheta[\delta+\epsilon]^{2} \nrightarrow 0,
$$

so that the KKS cosmological constant does not vanish point by point on moduli space.

\section{Outline of the Derivation}

We now provide an outline of the construction of the scattering amplitudes $\mathbf{A}_{I I}$ described in Section $\S 2$. In the Neveu-Schwarz-Ramond formulation of superstrings, the superstring action is given by

$$
\begin{aligned}
I_{m}\left(x^{\mu}, \psi_{ \pm}^{\mu} ; g_{m n}, \chi_{m}{ }^{\alpha}\right)= & \frac{1}{4 \pi} \int_{\Sigma} d^{2} z\left(\partial_{z} x^{\mu} \partial_{\bar{z}} x^{\mu}-\psi_{+}^{\mu} \partial_{\bar{z}} \psi_{+}^{\mu}-\psi_{-}^{\mu} \partial_{z} \psi_{-}^{\mu}\right. \\
& \left.+\chi_{\bar{z}}{ }^{+} \psi_{+}^{\mu} \partial_{z} x^{\mu}+\chi_{z}{ }^{-} \psi_{-} \partial_{\bar{z}} x^{\mu}-\frac{1}{2} \chi_{\bar{z}}{ }^{+} \chi_{z}{ }^{-} \psi_{+}^{\mu} \psi_{-}^{\mu}\right) .
\end{aligned}
$$

Here we have fixed a smooth surface $\Sigma$ of genus $h, g_{m n}$ is a metric on $\Sigma$, and $x^{\mu}, 0 \leq \mu \leq 9$, are scalar fields on $\Sigma$ which can be interpreted geometrically as a map from $\Sigma$ into 10-dimensional flat Minkowski space-time. The fields $\psi_{ \pm}^{\mu}$ and $\chi_{m}{ }^{\alpha}$ are respectively (anticommuting) Majorana-Weyl spinors and gravitino fields, defined with respect to a given spin structure $\delta$, so that $\psi_{ \pm} \in \Lambda^{ \pm \frac{1}{2}, 0}[\delta](\Sigma)$ and 
$\chi_{\bar{z}}{ }^{+} \in \Lambda^{0,1} \otimes \Lambda^{-\frac{1}{2}, 0}[\delta](\Sigma), \chi_{z}{ }^{-} \in \Lambda^{1,0} \otimes \Lambda^{0,-\frac{1}{2}}[\delta](\Sigma)$ if we view $\delta$ as a choice of a square root $\Lambda^{\frac{1}{2}, 0}[\delta](\Sigma)$ of the canonical bundle of $\Sigma$.

The sums over the fluctuating worldsheets spanned by evolving strings are realized by summing over all fields $x^{\mu}, g_{m n}, \psi_{ \pm}^{\mu}, \chi_{m}{ }^{\alpha}$. Without the spinor fields $\psi_{ \pm}^{\mu}$ and $\chi_{m}{ }^{\alpha}$, the action $I_{m}$ would reduce to the action for harmonic maps from $\Sigma$ in flat space-time, and its conformal invariance would clearly produce an integral over the moduli space $\mathcal{M}_{h}$ of Riemann surfaces of genus $h$. In the present superstring context, the metric $g_{m n}$ has been replaced by the "supergeometry" $\left(g_{m n}, \chi_{m}{ }^{\alpha}\right)$, and the action acquires a new symmetry, namely local supersymmetry. We discuss geometric aspects of this symmetry before returning to the evaluation of the sums over fluctuating worldsheets.

\subsection{Two-dimensional supergeometries and supermoduli.} The infinitesimal generator of a local supersymmetry is a spinor field $\delta \zeta^{\alpha}$, and its infinitesimal action on supergeometries is

$$
\delta e_{m}{ }^{a}=\delta \zeta \gamma^{a} \chi_{m}, \quad \delta \chi_{m}{ }^{\alpha}=-2 \nabla_{m} \delta \zeta^{\alpha},
$$

with similar actions on pairs $\left(x^{\mu}, \psi_{ \pm}^{\mu}\right)$. Here $e_{m}{ }^{a}$ is an orthonormal frame for the metric $g_{m n}=e_{m}{ }^{a} e_{n}{ }^{b} \delta_{a b}$. There is an evident similarity between local supersymmetry transformations and infinitesimal diffeomorphisms, which are generated by a vector field $\delta v^{n}$, and are given by $\delta e_{m}{ }^{a}=v^{n} \nabla_{n} e_{m}{ }^{a}+e_{n}{ }^{a} \nabla_{m} v^{n}, \delta \chi_{m}{ }^{\alpha}=\delta v^{n} \nabla_{n} \chi_{m}{ }^{\alpha}+\chi_{n}{ }^{\alpha} \nabla_{m} \delta v^{n}$. This similarity can be made more precise in the superspace formalism [38]. Let $s \Sigma$ be a supermanifold with $\Sigma$ as body, and local coordinates $\mathbf{z}=\left(z^{M}\right)=(z, \bar{z}, \theta, \bar{\theta})$, where $\theta, \bar{\theta}$ are anti-commuting. A supergeometry can then be identified with a superframe (or superzweibein) $E_{M}{ }^{A}$ and a $U(1)$ superconnection $\Omega_{M}$ satisfying the Wess-Zumino torsion constraints

$$
T_{a b}{ }^{c}=T_{\alpha \beta}{ }^{\gamma}=0, \quad T_{\alpha \beta}{ }^{c}=2\left(\gamma^{c}\right)_{\alpha \beta},
$$

where the torsion $T_{A B}{ }^{C}$ and curvature $R_{A B}$ of the superconnection $\Omega_{M}$ are defined by $\left[\mathcal{D}_{A}, \mathcal{D}_{B}\right]=T_{A B}{ }^{C} \mathcal{D}_{C}+i n R_{A B}$, and $\mathcal{D}_{A} V=E_{A}{ }^{M}\left(\partial_{M} V_{B}+\right.$ $i n \Omega_{M} V$ ) is the covariant derivative on fields $V$ of $\mathrm{U}(1)$ weight $n$. The group $s \operatorname{Diff}(\Sigma)$ acts on supergeometries by

$$
\delta E_{M}{ }^{B}=E_{M}^{A}\left(\mathcal{D}_{A} \delta V^{B}-\delta V^{C} T_{C A}{ }^{B}+\delta V^{C} \Omega_{C} E_{A}{ }^{B}\right) .
$$

The equivalence with the earlier definition of a supergeometry as $\left(g_{a b}, \chi_{a}{ }^{\alpha}\right)$ is obtained by putting the superframe $E_{M}{ }^{A}$ in the WessZumino gauge, where the frame components $E_{\mu}{ }^{\alpha}$ and $E_{\mu}{ }^{a}$ are required to satisfy $E_{\mu}{ }^{\alpha} \sim \delta_{\mu}{ }^{\alpha}+\theta^{\nu} e_{\nu \mu}^{* \alpha}, E_{\mu}{ }^{a} \sim \theta^{\nu} e_{\nu \mu}^{* * a}$ for some $e_{\nu \mu}^{* a}$ and $e_{\nu \mu}^{* * a}$ symmetric in $\nu$ and $\mu$. In such a gauge, the component $E_{m}{ }^{a}$ takes the form

$$
E_{m}{ }^{a}=e_{m}{ }^{a}+\theta \gamma^{a} \chi_{m}-\frac{i}{2} \theta \bar{\theta} e_{m}^{a} A
$$


with all other components of $E_{M}{ }^{A}$ and $\Omega_{M}$ expressible as well in terms of $e_{m}{ }^{a}, \chi_{m}$, and $A$. The auxiliary field $A$ can be set to 0 for all practical purposes, and we obtain in this manner the desired identification of the supergeometry $E_{M}{ }^{A}, \Omega_{M}$ with the pair $e_{m}{ }^{a}, \chi_{m}$. A vector field $\delta V^{M}$ in superspace can then be decomposed into components $\delta v^{m}$ and $\delta \zeta^{\alpha}$, and the corresponding superdiffeomorphisms decompose correspondingly into diffeomorphisms and local supersymmetry transformations. Similarly, super Weyl transformations can be defined which decompose into the standard Weyl transformations and the super Weyl transformations proper. The fields $x^{\mu}$ and $\psi_{ \pm}^{\mu}$ can also be grouped into a scalar superfield $X^{\mu}(z, \theta, \bar{\theta})=x^{\mu}+\theta \psi_{+}^{\mu}+\bar{\theta} \psi_{-}^{\mu}$. In Wess-Zumino gauge, the covariant derivative of a superfield $V(z, \theta, \bar{\theta})=V_{0}+\theta V_{+}+\bar{\theta} V_{-}$of $\mathrm{U}(1)$ weight $n$ becomes [28],

$$
\begin{aligned}
\mathcal{D}_{-}^{(n)} V= & V_{-}+\bar{\theta}\left(\partial_{\bar{z}} V_{0}+\frac{1}{2} \chi_{\bar{z}}{ }^{+} V_{+}\right) \\
& -\theta \bar{\theta}\left(\partial_{\bar{z}} V_{+}+\frac{1}{2} \chi_{\bar{z}}{ }^{+} \partial_{z} V_{0}+n \partial_{z} \chi_{\bar{z}}{ }^{+} V_{0}-\frac{1}{4} \chi_{\bar{z}}{ }^{+} \chi_{z}{ }^{-} V_{-}\right) .
\end{aligned}
$$

Introducing the measure $d^{2 \mid 2} \mathbf{z}=d^{2} z d \theta d \bar{\theta}$ and the volume element $E(\mathbf{z})=\operatorname{sdet} E_{M}{ }^{A}=\left(\operatorname{det} e_{m}{ }^{a}\right)\left(1+\frac{1}{4} \theta \bar{\theta} \chi_{\bar{z}}{ }^{+} \chi_{z^{-}}\right)$, the action $I_{m}$ can be expressed in the following manifestly supersymmetric and super Weyl invariant form

$$
I_{m}\left(E_{M}^{A}, X^{\mu}\right)=\frac{1}{4 \pi} \int d^{2 \mid 2} \mathbf{z} E(\mathbf{z}, \overline{\mathbf{z}}) \mathcal{D}_{+} X^{\mu} \mathcal{D}_{-} X^{\mu} .
$$

- Associated to each supergeometry is a notion of superholomorphicity. In the superspace formalism, we can define a supercomplex structure $J_{M}^{N}$ by $[\mathbf{2 3}]$

$$
J_{M}^{N}=E_{M}^{a} \epsilon_{a}^{b} E_{b}{ }^{N}+E_{M}^{\alpha}\left(\gamma_{5}\right)_{\alpha}^{\beta} E_{\beta}{ }^{N}
$$

which satisfies $J_{M}^{N} J_{N}^{P}=-\delta_{M}{ }^{N}$ and the integrability condition $d \zeta^{M} \equiv$ $0\left(\bmod \zeta^{N}\right)$, where $\zeta^{M} \equiv d z^{M}-i d z^{N} J_{N}^{M}$. A scalar function $f(z, \theta)$ is then defined to be superholomorphic if $J_{M}{ }^{N} \mathcal{D}_{N} f=0$, or equivalently $\mathcal{D}_{-} f=0$. More generally, a field $\hat{\omega}(z, \theta)$ on $s \mathcal{M}$ of $\mathrm{U}(1)$ weight $n$ is said to be superholomorphic if

$$
\mathcal{D}_{-}^{(n)} \hat{\omega}=0
$$

where $\mathcal{D}_{-}^{(n)}$ is the covariant derivative on fields of weight $n$ with respect to the given supergeometry. In particular, for a form $\hat{\omega}$ of $\mathrm{U}(1)$ weight $1 / 2$ of the form $\hat{\omega}(z, \theta)$ as $\hat{\omega}(z, \theta)=\omega_{0}+\theta \omega_{+}$, the superholomorphicity condition is equivalent to the following system of partial differential equations on $\Sigma$

$$
\partial_{\bar{z}} \omega_{0}+\frac{1}{2} \chi_{\bar{z}}{ }^{+} \omega_{+}=0, \quad \partial_{\bar{z}} \omega_{+}+\frac{1}{2} \partial_{z}\left(\chi_{\bar{z}}{ }^{+} \omega_{0}\right)=0
$$


- A key property of supergeometries $\left(g_{m n}, \chi_{m}{ }^{\alpha}\right)$ defined by an even spin structure $\delta$ is that, generically, there exists a unique basis of superholomorphic forms $\hat{\omega}_{I}$ of $\mathrm{U}(1)$ weight $1 / 2$ dual to the $A_{I}$ cycles, and hence a super period matrix $\hat{\Omega}_{I J}$ can be defined by

$$
\oint_{A_{J}} \hat{\omega}_{J}=\delta_{I J}, \quad \oint_{B_{J}} \hat{\omega}_{J}=\hat{\Omega}_{I J} .
$$

Here the integral over a cycle $C$ of a form $\hat{\omega}=\omega_{0}+\theta \omega_{+}$of $\mathrm{U}(1)$ weight $1 / 2$ is defined by $\oint_{C} \hat{\omega}=\oint_{C}\left(d z \omega_{+}-\frac{1}{2} d \bar{z} \chi_{\bar{z}}{ }^{+} \omega_{0}\right)$. Explicitly, $\hat{\Omega}_{I J}$ and $\Omega_{I J}$ can be determined from each other by the following equation

$$
\hat{\Omega}_{I J}=\Omega_{I J}-\frac{i}{8 \pi} \iint d^{2} y d^{2} x \omega_{I}(x) \chi_{\bar{x}}{ }^{+} \hat{S}_{\delta}(x, y) \chi_{\bar{y}}{ }^{+} \omega_{J}(y),
$$

where $\omega_{I}$ is a basis of holomorphic 1-forms with respect to the complex structure defined by $g_{m n}$, and $\hat{S}_{\delta}(x, y)$ is the modification of the Szegö kernel of $g_{m n}$ by

$$
\begin{aligned}
& \hat{S}_{\delta}(z, w)=S_{\delta}(z, w) \\
& -\frac{i}{16 \pi^{2}} \iint d^{2} u d^{2} v S_{\delta}(z, u) \chi_{\bar{u}}{ }^{+} \partial_{u} \partial_{v} \ln E(u, v) \chi_{\bar{v}}{ }^{+} \hat{S}_{\delta}(v, w),
\end{aligned}
$$

with $E(u, v)$ the prime form. By construction, the super period matrix $\hat{\Omega}_{I J}$ is invariant under all symmetry transformations, including supersymmetry. In genus $h=2$, the super period matrix $\hat{\Omega}_{I J}$ is always well-defined for even spin structures.

- We come now to the essential relation between superholomorphicity and holomorphicity that underlies our derivation of superstring scattering amplitudes. First, we note that there can be no intrinsic relation between the superholomorphicity of a form $\hat{\omega}=\omega_{0}+\theta \omega_{+}$and the holomorphicity of its components, if the latter notion of holomorphicity is taken with respect to the metric $g_{m n}$. This is simply because the conformal class of the metric $g_{m n}$ is not left invariant under supersymmetry transformations. The only candidate for a supersymmetric substitute is the super period matrix $\hat{\Omega}_{I J}$.

Thus, we choose a metric $\hat{g}_{m n}$ whose period matrix is $\hat{\Omega}_{I J}$. Such a metric is only determined up to diffeomorphisms, and the relation we need between superholomorphicity and holomorphicity with respect to the metric $\hat{g}_{m n}$ has to take into account this gauge choice. Furthermore, because of the deformation of complex structure from $g_{m n}$ to $\hat{g}_{m n}$, the forms $\omega_{0}$ and $\omega_{+}$are no longer pure $(p, 0)$-forms with respect to $\hat{g}_{m n}$, so they cannot possibly be holomorphic. The guiding principle is that the $\theta$-component of a superholomorphic form with respect to the supergeometry $\left(g_{m n}, \chi_{m}{ }^{\alpha}\right)$ is a holomorphic form with respect to $\hat{g}_{m n}$, up to a de Rham exact differential. We provide below some explicit examples of this relation between holomorphicity and superholomorphicity in the case of genus $h=2$. In this case, the calculations are relatively simpler 
because there are only 2 odd supermoduli $\zeta^{\alpha}$, and perturbation theory need only be worked out to first even order $\zeta^{1} \zeta^{2}$. Similar formulas can be expected to hold in higher genus.

(a) Let $\hat{\omega}(z, \theta)$ be a weight $1 / 2$ superholomorphic form with respect to $\left(g_{m n}, \chi_{m}{ }^{\alpha}\right)$. Let the supervolume form $d^{2 \mid 2} \mathbf{z} E(\mathbf{z}, \overline{\mathbf{z}})$ be chirally split as $d^{2 \mid 2} \mathbf{z} E(\mathbf{z}, \overline{\mathbf{z}})=d \bar{\theta} \wedge e^{\bar{z}} \wedge d \theta \wedge e^{z}$ with $e^{z}=d z-\frac{1}{2} \theta \chi_{\bar{z}}{ }^{+} d \bar{z}$. (c.f. [23], eqs. (3.32)-(3.33)). Then

$$
\int d \theta e^{z} \hat{\omega}=\omega(z)+d \lambda(z)
$$

where $\omega(z)$ is a holomorphic $(1,0)$-form with respect to $\hat{g}_{m n}$, and $\lambda(z)$ is a $C^{\infty}$ scalar function. Under changes of metrics $\hat{g}_{m n}, \lambda$ changes by $\delta \lambda=-\delta v^{z} \omega(z)$.

(b) Let $\mathcal{E}_{\delta}(\mathbf{z}, \mathbf{w})$ be the super prime form (see $[\mathbf{2 8}]$ for the definition). Then there exists a scalar function $\hat{f}_{0}(z, w)$ so that

$$
\begin{aligned}
& \int d \theta_{z_{i}} \int d \theta_{z_{j}} e^{z_{i}} e^{z_{j}} \mathcal{D}_{+}^{\mathbf{z}_{i}} \mathcal{D}_{+}^{\mathbf{z}_{j}} \ln \mathcal{E}_{\delta}\left(\mathbf{z}_{i}, \mathbf{z}_{j}\right) \\
&=d z_{i} \wedge d z_{j} \partial_{z_{i}} \partial_{z_{j}} \ln E\left(z_{i}, z_{j}\right)-d_{i} d_{j} \hat{f}_{0}\left(z_{i}, z_{j}\right),
\end{aligned}
$$

up to Dirac measures supported at coincident points. By the cancelled propagator argument, amounting to an analytic continuation in $s_{i j}=-2 k_{i} \cdot k_{j}[\mathbf{3 2}]$, such Dirac measures can always be dropped in presence of the factor $\prod_{i<j} E\left(z_{i}, z_{j}\right)^{k_{i} \cdot k_{j}}$. Thus, up to exact de Rham differentials, the highly non-holomorphic term $\mathcal{D}_{+}^{\mathbf{z}} \mathcal{D}_{+}^{\mathbf{w}} \ln \mathcal{E}_{\delta}(\mathbf{z}, \mathbf{w})$ reduces to the holomorphic function $\partial_{z} \partial_{w} \ln E(z, w)$.

(c) The relation between holomorphicity and superholomorphicity leads to many new holomorphic forms on moduli space, the existence of which may not have been suspected otherwise. For example, if we write $\hat{\omega}_{I}=\omega_{I 0}+\theta \omega_{I+}$, and let $\lambda_{I}$ be the scalar function defined up to a constant by $\hat{\omega}_{I 0}=\omega_{I}(z)+d \lambda_{I}(z)$, then the expression

$$
\Pi_{I J}^{(1)}(z)=\omega_{I}(z) \lambda_{J}(z)-\omega_{J}(z) \lambda_{I}(z)-\hat{\omega}_{I 0}(z) \hat{\omega}_{J 0}(z)
$$

is a holomorphic form. Many other holomorphic forms in more variables can be constructed in the same manner from components of superholomorphic forms.

(d) In superstring perturbation theory, it is necessary to consider superholomorphic forms with certain non-trivial monodromies, as in (2.2). Here the relation between superholomorphicity and holomorphicity has been established so far only through involved explicit calculations, for the specific superholomorphic forms arising from correlation functions of scalar superfields. The relation between the holomorphic form $\mathcal{H}$ and the combination $\sum_{\delta} \epsilon_{\delta} \mathcal{B}[\delta]$ described in Section $\S 2$ is a prime example. 
- The supermoduli space of the surface $\Sigma$ is defined to be

$$
s \mathcal{M}_{h}=\left\{\left(g_{m n}, \chi_{m}{ }^{\alpha}\right)\right\} /\{\text { symmetries }\},
$$

where the symmetries are generated by Weyl, super Weyl, diffeomorphisms, and supersymmetry transformations. The tangent space $T\left(s \mathcal{M}_{h}\right)$ to $s \mathcal{M}_{h}$ decomposes as $\left\{\delta g_{m n}\right\} \oplus\left\{\delta \chi_{m}{ }^{\alpha}\right\}$. In local complex coordinates $z, \bar{z}$ for the metric $g_{m n}$, we may set $\delta g_{\bar{z} z}=0$ and $\delta \chi_{\bar{z}}{ }^{-}=$ $\delta \chi_{z}{ }^{+}=0$ by Weyl and super Weyl transformations. The dimension of the remaining modes $\delta g_{z z}$ and $\delta \chi_{\bar{z}}{ }^{+}$in $T\left(s \mathcal{M}_{h}\right)$ after diffeomorphisms and supersymmetry transformations can be easily determined by their values at $\chi=0$, where they are given respectively by the codimensions of the $\bar{\partial}$ operators on tensors of $U(1)$ weights 2 and $3 / 2$ respectively. By the Riemann-Roch theorem, we obtain

$$
\operatorname{dim}\left(s \mathcal{M}_{h}\right)= \begin{cases}(0 \mid 0), & \text { if } h=0 \\ (1 \mid 0)_{e} \text { or }(1 \mid 1)_{0}, & \text { if } h=1 \\ (3 h-3 \mid 2 h-2), & \text { if } h \geq 2,\end{cases}
$$

where the dimensions indicated for genus 1 depend on whether the spin structure $\delta$ is even or odd, as indicated by the indices $e$ or $o$.

3.2. Functional integrals. We return to the derivation of the superstring scattering amplitudes. We start from sums over fluctuating worldsheets given by the following functional integrals

$$
\begin{aligned}
\mathbf{A}[\delta]=\int D E_{M}{ }^{A} D \Omega_{M} \delta( & T) \\
& \times \int \prod_{i=1}^{N} d^{2 \mid 2} \mathbf{z}_{i} E\left(\mathbf{z}_{i}\right) \\
& \times \int X^{\mu} e^{-I_{m}} \prod_{i=1}^{N} V\left(\mathbf{z}_{i}, \overline{\mathbf{z}}_{i} ; \epsilon_{i}, \bar{\epsilon}_{i}, k_{i}\right)
\end{aligned}
$$

where $V\left(\mathbf{z}_{i}, \overline{\mathbf{z}}_{i} ; \epsilon_{i}, \bar{\epsilon}_{i}, k_{i}\right)=\exp \left(i k_{i}^{\mu} X^{\mu}\left(\mathbf{z}_{i}\right)+\epsilon_{i}^{\mu} \mathcal{D}_{+} X^{\mu}+\bar{\epsilon}_{i}^{\mu} \mathcal{D}_{-} X^{\mu}\right), k^{2}=$ $k \cdot \epsilon=k \cdot \bar{\epsilon}=0$, is the generating vertex for the graviton multiplet [39]. Factoring out all symmetries reduces these functional integrals to an integral over supermoduli space ([23], eq. (3.143))

$$
\begin{aligned}
\mathbf{A}[\delta]=\int\left|\prod_{A} d m^{A}\right|^{2} & \int \prod_{i=1}^{N} d^{2 \mid 2} \mathbf{z}_{i} E\left(\mathbf{z}_{i}\right) \int D\left(B \bar{B} C \bar{C} X^{\mu}\right) e^{-I_{m}-I_{g h}} \\
\times & \left|\prod_{A} \delta\left(\left\langle H_{A} \mid B\right\rangle\right)\right|^{2} V\left(\mathbf{z}_{i}, \overline{\mathbf{z}}_{i} ; \epsilon_{i}, \bar{\epsilon}_{i}, k_{i}\right) .
\end{aligned}
$$

Here $m^{A}$ are $(3 h-3 \mid 2 h-2)$ local complex parameters for a slice $\hat{\mathcal{S}}$ for supermoduli space,

$$
\left(H_{A}\right)_{-}{ }^{z}=(-)^{A(M+1)} E_{-}{ }^{M} \frac{\partial E_{M}^{z}}{\partial m^{A}}
$$


are the super Beltrami differentials tangent to the gauge slice $\hat{\mathcal{S}}$, and the Faddeev-Popov determinants of the gauge-fixing procedure have been encoded in an integration over the superghost fields $B=\beta+\theta b$, $C=c+\theta \gamma$ of $U(1)$ weights $3 / 2$ and -1 respectively with action $I_{g h}=$ $\frac{1}{2 \pi} \int d^{2 \mid 2} \mathbf{z} E\left(B \mathcal{D}_{-} C+\bar{B} \mathcal{D}_{+} \bar{C}\right)$. In components, the superghost action can be expressed as

$$
I_{g h}=\int d^{2} z\left\{b \partial_{\bar{z}} c+\beta \partial_{\bar{z}} \gamma+\chi_{\bar{z}}{ }^{+} S_{g h}+c . c .\right\},
$$

where $S_{g h}=\frac{1}{2} b \gamma-\frac{3}{2} \beta \partial_{z} c-\left(\partial_{z} \beta\right) c$ is the ghost supercurrent.

- The integrals $\mathbf{A}[\delta]$ are only a preliminary step in constructing the superstring scattering amplitudes. To obtain these, one has to identify in $\mathbf{A}[\delta]$ the contributions of each chiral sector, and sum these contributions over $\delta$, with suitable phases $\epsilon_{\delta}$ so as to insure modular invariance. The chiral sector corresponds to the correct degrees of freedom of the Minkowski formalism, and the summation over spin structures is the Gliozzi-Scherk-Olive projection, necessary for eliminating tachyons and insuring space-time supersymmetry.

The identification of the contributions of each chiral sector is provided by the chiral splitting theorem of [28], which asserts that

$$
\begin{aligned}
& \int D X^{\mu} \prod_{i=1}^{N} e^{-I_{m}} V\left(\mathbf{z}_{i}, \overline{\mathbf{z}}_{i} ; \epsilon_{i}, \bar{\epsilon}_{i}, k_{i}\right) \\
& =\int d p_{I}^{\mu}\left|\left\langle Q\left(p_{I}\right) \exp \left(\frac{1}{2 \pi} \int \chi S_{m}\right) \prod_{i=1}^{N} W\left(\mathbf{z}_{i} ; \epsilon_{i}, k_{i}\right)\right\rangle_{+}\right|^{2}
\end{aligned}
$$

where $Q\left(p_{I}\right)=\exp \left\{i p_{I}^{\mu} \oint_{B_{I}} d z \partial_{z} x_{+}^{\mu}(z)\right\}, W(\mathbf{z} ; \epsilon, k)$ is the chiral generating vertex given by

$$
W(\mathbf{z} ; \epsilon, k)=\exp \left\{i k^{\mu}\left(x_{+}^{\mu}+\theta \psi_{+}^{\mu}\right)(z)+\epsilon^{\mu}\left(\psi_{+}+\theta \partial_{z} x_{+}^{\mu}\right)(z)\right\} .
$$

The expectation value $\langle\cdot\rangle_{+}$is taken with respect to an effective bosonic chiral field $x_{+}^{\mu}(z)$ with propagator $\left\langle x_{+}^{\mu}(z) x_{+}^{\nu}(w)\right\rangle=-\delta^{\mu \nu} \ln E(z, w)$, and a fermionic field $\psi_{+}^{\mu}(z)$ with propagator $\left\langle\psi_{+}^{\mu}(z) \psi_{+}^{\nu}(w)\right\rangle=-\delta^{\mu \nu} S_{\delta}(z, w)$, where $S_{\delta}(z, w)$ is the Szegö kernel. The expression $S_{m}$ is the effective matter supercurrent $S_{m}=-\frac{1}{2} \psi_{+}^{\mu} \partial_{z} x_{+}^{\mu}$. The point of this formula is that, by introducing the parameters $p_{I}^{\mu}$, the real bosonic field $x^{\mu}(z)$ has been replaced by a chiral field $x_{+}^{\mu}$, and all terms mixing opposite chiralities such as $\chi_{\bar{z}}{ }^{+}, \psi_{+}^{\mu}$ with $\chi_{z}{ }^{-}, \psi_{-}^{\mu}$ have cancelled out. Physically, as in the case of the bosonic string discussed in [11], the parameters $p_{I}^{\mu}$, $1 \leq I \leq h, 0 \leq \mu \leq 9$, can be interpreted as internal loop momenta.

- We also need to split chirally the super volume form $d^{2 \mid 2} \mathbf{z} E(\mathbf{z}, \overline{\mathbf{z}})$ on the superworldsheet. As we saw earlier, from [23], eqs. (3.32)-(3.33), we have $d^{2 \mid 2} \mathbf{z} E(\mathbf{z}, \overline{\mathbf{z}})=d \bar{\theta} \wedge e^{\bar{z}} \wedge d \theta \wedge e^{z}$ with $e^{z}=d z-\frac{1}{2} \theta \chi_{\bar{z}}{ }^{+} d \bar{z}$. If we 
now let

$$
\begin{aligned}
\mathcal{V}(z ; \epsilon, k) & =\int d \theta e^{z} W(\mathbf{z} ; \epsilon, k) \\
& =\epsilon^{\mu}\left\{\left(\partial_{z} x_{+}^{\mu}-i k^{\nu} \psi_{+}^{\mu} \psi_{+}^{\nu}\right) d z-\frac{1}{2} d \bar{z} \chi_{\bar{z}}^{+} \psi_{+}^{\mu}\right\} e^{i k \cdot x_{+}(z)}
\end{aligned}
$$

we can write

$$
\begin{aligned}
\mathbf{A}[\delta]=\int d p_{I}^{\mu} \int_{\mathcal{S}} \mid \prod_{A=1}^{(3 h-3 \mid 2 h-2)} d m^{A}\left\langle\prod_{A} \delta\left(H_{A} \mid B\right) Q\left(p_{I}\right)\right. \\
\\
\left.\quad \times \exp \left\{\frac{1}{2 \pi} \int \chi S\right\} \prod_{j=1}^{N} \mathcal{V}_{j}\right\rangle\left.\right|^{2} .
\end{aligned}
$$

Here the expectation value is with respect to all chiral fields $x_{+}^{\mu}, \psi_{+}^{\mu}$, $b, c, \beta, \gamma$, and $S=S_{m}+S_{g h}$ is the total supercurrent, incorporating the effective matter supercurrent $S_{m}$ as well as the ghost supercurrent $S_{g h}$ from the superghost action $I_{g h}$. Naively, after implementation of the Gliozzi-Scherk-Olive projection, the scattering amplitude $\mathbf{A}_{I I}$ should be given by

$$
\begin{aligned}
\mathbf{A}_{I I}=\int d p_{I}^{\mu} \int_{\mathcal{S}} \prod_{A=1}^{(3 h-3 \mid 2 h-2)} d m^{A} \sum_{\delta} \epsilon_{\delta}\left\langle\prod_{A} \delta\left(H_{A} \mid B\right) Q\left(p_{I}\right)\right. \\
\\
\left.\times \exp \left\{\frac{1}{2 \pi} \int \chi S\right\} \prod_{j=1}^{N} \mathcal{V}_{j}\right\rangle\left.\right|^{2} .
\end{aligned}
$$

We should stress that all complex coordinates and correlation functions are at this time written with respect to the metric $g_{m n}$ from the slice $\hat{\mathcal{S}}$.

So far, the gauge-fixed formula (3.26) holds for an arbitrary choice of $(3 h-3 \mid 2 h-2)$-dimensional slice $\hat{\mathcal{S}}$ in the space of supergeometries. The issue is whether the odd supermoduli $d m^{\alpha}$ can be integrated out to produce a global form over moduli space. Perhaps surprisingly, this turns out not to be the case with the naive projections used in the early 1980s, and the origin of the problem has been somewhat of a mystery ever since $[\mathbf{1 1}, \mathbf{1 2}]$. We discuss it and its resolution in the next section.

3.3. Deformation of complex structures. The above FaddeevPopov type gauge-fixing procedure shows that, upon cancellation of all anomalies, the sums over all supergeometries can be reduced to sums over supermoduli space, after factoring out all symmetries. The new difficulty peculiar to superstring perturbation theory is that the superstring amplitudes have to be expressed as sums over moduli space and not as sums over supermoduli space. To go from supermoduli to moduli, a correct structure for supermoduli space as a fibration over moduli space has to be identified, and the odd supermoduli degrees of freedom 
integrated out. This deceptively simple problem has to be approached with some care.

- The projection $\left(g_{m n}, \chi_{m}{ }^{\alpha}\right) \rightarrow g_{m n}$ from supergeometries to geometries seems a natural candidate for constructing such a fibration. However, it is not well-defined as a projection from supermoduli space to moduli space, as supergeometries equivalent under supersymmetries do not project to geometries equivalent under diffeomorphisms and Weyl transformations

$$
\begin{array}{ccc}
\left(g_{m n}, \chi_{m}{ }^{\alpha}\right) & \sim & \left(g_{m n}+\delta g_{m n}, \chi_{m}{ }^{\alpha}+\delta \chi_{m}{ }^{\alpha}\right) \\
g_{m n} & \nsim & \downarrow \\
\downarrow & g_{m n}+\delta g_{m n}
\end{array} .
$$

The only alternative is to rely instead on the super period matrix $\hat{\Omega}$ and the projection

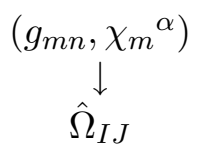

which is invariant under supersymmetry and does descend to the complement of a lower-dimensional subvariety in supermoduli space. We develop now the gauge-fixing procedure based on this projection.

- As in our earlier discussion of the relation between superholomorphicity and holomorphicity with respect to $\hat{\Omega}_{I J}$, the projection $\left(g_{m n}, \chi_{m}{ }^{\alpha}\right) \rightarrow \hat{\Omega}_{I J}$ has to be supplemented by a choice of metric $\hat{g}_{m n}$ whose period matrix is $\hat{\Omega}_{I J}$. There is no canonical $\hat{g}_{m n}$, and different choices of $\hat{g}_{m n}$ are related infinitesimally by $\delta \hat{g}_{m n}=\nabla_{m} \delta v_{n}+\nabla_{n} \delta v_{n}$, where $\delta v^{n}$ is a smooth vector field on $\Sigma$. In genus $h=2$, the deformation from $\hat{g}_{m n}$ to $g_{m n}$ is only of first order in $\zeta^{1} \zeta^{2}$, and we may define its Beltrami differential $\hat{\mu}_{\bar{z}}{ }^{z}=\frac{1}{2} \hat{g}^{z \bar{z}} g_{\bar{z} \bar{z}}$ in local holomorphic coordinates for $\hat{g}_{m n}$. Then $\hat{\mu}_{\bar{z}}{ }^{z}$ is defined by the condition

$$
i \int_{\Sigma} \omega_{I} \omega_{J} \hat{\mu}_{\bar{z}}^{z} d^{2} z=\Omega_{I J}-\hat{\Omega}_{I J} .
$$

This equation determines $\hat{\mu}_{\bar{z}}^{z}$ only up to a gauge choice of $\delta \hat{\mu}_{\bar{z}}{ }^{z}=\partial_{\bar{z}} \delta v^{z}$.

A choice of metrics is necessary because the correlation functions of conformal and superconformal field theories require an underlying geometry or supergeometry, and not just an equivalence class under diffeomorphisms and/or supersymmetry transformations. It will be an important check of the consistency of our gauge-fixing procedure for superstring amplitudes that, after integration over all insertion points, the final amplitude is independent of the choice of $\hat{\mu}_{\bar{z}}{ }^{z}$.

- We can construct a slice $\hat{\mathcal{S}}$ for supermoduli space which fibers over the period matrices $\hat{\Omega}$ as follows. Let $\hat{\Omega}_{I J}, 1 \leq I \leq J \leq 2$, be the 3 local holomorphic coordinates for moduli space, and choose a 3 -dimensional 
slice $\hat{S}$ of frames $\hat{e}_{m}{ }^{a}$ whose period matrices are the matrices $\hat{\Omega}_{I J}$. For each of these frames $\hat{e}_{m}{ }^{a}$, choose 2 generic gravitino sections $\hat{\chi}_{\alpha}, \alpha=$ 1,2 , and set $\hat{\chi}=\sum_{\alpha=1}^{2} \zeta^{\alpha} \hat{\chi}_{\alpha}$, where $\zeta^{\alpha}$ are 2 anticommuting parameters. We can choose next a $(3 \mid 2)$-dimensional slice of supergeometries $\left(e_{m}{ }^{a}, \chi\right)$ whose period matrices $\Omega_{I J}$ and $\hat{\Omega}_{I J}$ satisfy the equation (3.12). This can clearly be done, because $\Omega_{I J}$ and $\hat{\Omega}_{I J}$ differ by terms of order $O\left(\zeta^{1} \zeta^{2}\right)$, and thus gravitino sections $\hat{\chi}_{\alpha}$ with respect to $\hat{e}_{m}{ }^{a}$ can be considered as gravitino sections $\chi_{\alpha}$ with respect to $e_{m}{ }^{a}$.

There are three significant complications in this gauge-fixing procedure, compared to the earlier one based on the simpler but ill-behaved projection $\left(g_{m n}, \chi_{m}{ }^{\alpha}\right) \rightarrow g_{m n}$ :

(a) The first is that the Beltrami superdifferentials $H_{A}=\bar{\theta}\left(\hat{\mu}_{A}-\right.$ $\left.\theta \chi_{A}\right)$ defined by the slice $\hat{\mathcal{S}}$ have components $\hat{\mu}_{A}$ and $\nu_{A}$ which are both non-vanishing, unlike in the earlier case where one of the components $\hat{\mu}_{A}$ or $\nu_{A}$ is always 0 . This reflects the fact that, to maintain $\hat{\Omega}_{I J}$ fixed, both $g_{m n}$ and $\chi_{m}{ }^{\alpha}$ have to be deformed simultaneously.

(b) The second is that the correlation functions of the underlying conformal field theories are expressed in the background of the metric $g_{m n}$. To re-express them in the background of the metric $\hat{g}_{m n}$, we need to carry out a deformation of complex structures, and hence an insertion of the stress tensor $T(z)$.

(c) The third is that the vertex operators $\mathcal{V}$ have to be deformed as well. This produces new vertex operators

$$
\mathcal{V}(z)=\mathcal{V}^{(0)}(z)+\mathcal{V}^{(1)}(z)+\mathcal{V}^{(2)}(z)
$$

where $\mathcal{V}^{(0)}$ is the naive vertex operator of $(2.6)$, and $\mathcal{V}^{(1)}, \mathcal{V}^{(2)}$ are deformation corrections.

Taking all these points into account, we obtain the following first formula for the gauge-fixed amplitude,

$$
\mathbf{A}[\delta]=\int d p_{I}^{\mu} \int_{\mathcal{M}_{2} \times \Sigma^{N}}\left|\mathcal{B}[\delta]\left(z_{i} ; k_{i}, \epsilon_{i} ; p_{I}^{\mu}\right)\right|^{2}
$$

where the chiral forms $\mathcal{B}[\delta]\left(z_{i} ; k_{i}, \epsilon_{i} ; p_{I}^{\mu}\right)$ are given by

$$
\begin{aligned}
\mathcal{B}[\delta]\left(z_{i} ; k_{i}, \epsilon_{i} ; p_{I}^{\mu}\right)= & \prod_{I \leq J} d \hat{\Omega}_{I J} \int \prod_{\alpha=1,2} d \zeta^{\alpha} \frac{\left\langle\prod_{a} b\left(p_{a}\right) \prod_{\alpha} \delta\left(\beta\left(q_{\alpha}\right)\right)\right\rangle}{\operatorname{det} \Phi_{I J+}\left(p_{a}\right) \cdot \operatorname{det}\left\langle H_{\alpha} \mid \Phi_{\beta}^{*}\right\rangle} \\
& \times\left\langle Q\left(p_{I}\right) \exp \left\{\frac{1}{2 \pi} \int(\chi(z) S(z)+\hat{\mu}(z) T(z))\right\} \prod_{j=1}^{N} \mathcal{V}_{j}\right\rangle .
\end{aligned}
$$

Here $p_{a}, 1 \leq a \leq 3$, and $q_{\alpha}, 1 \leq \alpha \leq 2$, are two sets of arbitrary auxiliary points on $\Sigma, \Phi_{I J}(z, \theta)=\Phi_{I J 0}+\theta \Phi_{I J+}$ is the basis of superholomorphic differentials of weight $3 / 2$ defined by $-\frac{i}{2}\left(\hat{\omega}_{I} \mathcal{D}_{+} \hat{\omega}_{J}+\hat{\omega}_{I} \mathcal{D}_{+} \hat{\omega}_{J}\right), \Phi_{\beta}^{*}=$ $\Phi_{\beta 0}+\theta \Phi_{\beta+}^{*}$ is another basis of superholomorphic differentials of weight 
$3 / 2$ normalized by $\Phi_{\beta 0}^{*}\left(q_{\alpha}\right)=\delta_{\alpha \beta}$ and $\Phi_{\beta+}^{*}\left(p_{a}\right)=0$, and $S(z)$ is the supercurrent.

The three points which we stressed above are reflected in the appearance in this formula of the full vertex operator $\mathcal{V}\left(z_{i}\right)$, of the finitedimensional determinants $\Phi_{I J+}\left(p_{a}\right)$ and $\left\langle H_{\alpha} \mid \Phi_{\beta}^{*}\right\rangle$ corresponding to the gauge slice constructed, and of the insertion of the stress tensor $T_{z z}$ implementing the deformation of complex structures.

At this moment, after the deformation of complex structures has been carried, all correlation functions in $\mathcal{B}[\delta]\left(z_{i} ; k_{i}, \epsilon_{i} ; p_{I}^{\mu}\right)$ are expressed with respect to the metric $\hat{g}_{m n}$. The metric $g_{m n}$ and its period matrix no longer enter the picture, and we can now just denote $\hat{\Omega}_{I J}$ by $\Omega_{I J}$ for simplicity.

3.4. Gauge slice independence. We derive next explicit formulas for $\mathcal{B}[\delta]\left(z_{i} ; k_{i}, \epsilon_{i} ; p_{I}^{\mu}\right)$. In the process, we establish the independence of the amplitudes from all choices entering the gauge-fixing process. This also facilitates later explicit evaluations, since we shall be free to make convenient choices for different calculations.

- We begin with the 0-point function, in which case the dependence of $\mathcal{B}[\delta]$ on the external momenta is trivial, and $\mathcal{B}[\delta]$ reduces essentially to the measure $d \mu_{2}[\delta]$,

$$
\mathcal{B}[\delta]\left(p_{I}^{\mu}\right)=\exp \left(i \pi p_{I}^{\mu} \Omega_{I J} p_{J}^{\mu}\right) d \mu_{2}[\delta](\Omega), \quad N=0,
$$

and $d \mu_{2}[\delta]$ is itself given by

$$
\begin{aligned}
& d \mu_{2}[\delta](\Omega)=\frac{\left\langle\prod_{a} b\left(p_{a}\right) \prod_{\alpha} \delta\left(\beta\left(q_{\alpha}\right)\right)\right\rangle}{\operatorname{det} \Phi_{I J+}\left(p_{a}\right) \cdot \operatorname{det}\left\langle H_{\alpha} \mid \Phi_{\beta}^{*}\right\rangle} \\
& \times\left\{1-\frac{1}{8 \pi^{2}} \int d^{2} z \chi_{\bar{z}}{ }^{+} \int d^{2} w \chi_{\bar{w}}{ }^{+}\langle S(z) S(w)\rangle\right. \\
& \left.+\frac{1}{2} \int d^{2} z \hat{\mu}_{\bar{z}}^{z}\langle T(z)\rangle\right\} \text {. }
\end{aligned}
$$

The correlation functions of the supercurrent and stress tensor can now be evaluated in terms of prime forms and Green's functions. This gives the expression (2.8)-(2.12) for $d \mu_{2}[\delta]$ described earlier. The independence of gauge choices is then obtained by showing that the variations of $d \mu_{2}[\delta]$ under changes of $\chi_{\alpha}(z)$ as well as $\mu(z)$ vanish point by point on the moduli space $\mathcal{M}_{2}$. The case of $\chi_{\alpha}(z)=\delta\left(z, x_{\alpha}\right)$ is particularly convenient. In this case, $d \mu_{2}[\delta]$ reduces to the expression (2.17). This expression can be independently verified to be independent of all points $p_{a}, q_{\alpha}$, and $x_{\alpha}$. Note that the Beltrami differential $\hat{\mu}(z)$ has cancelled out, so we have manifest independence from the choice of metrics $\hat{g}_{m n}$.

- Next, we show the gauge slice independence of the $N$-point function. Since $d \mu_{2}[\delta]$ has been shown to be gauge slice independent, and since the factor $\left\langle Q\left(p_{I}\right) \prod_{i=1}^{N} \mathcal{V}\left(z_{i}\right)\right\rangle$ does not depend on any gauge choice, 
the term $\mathcal{B}[\delta]^{(d)}$ is gauge-slice independent. As for the term $\mathcal{B}^{(c)}$, we can show that it transforms as

$$
\delta \mathcal{B}[\delta]^{(c)}=\sum_{i=1}^{N} d_{i} \mathcal{R}_{i}[\delta],
$$

with $\mathcal{R}_{i}[\delta]$ given respectively by

$$
\begin{aligned}
\mathcal{R}_{i}[\delta]=- & \delta v^{z_{i}}\left\langle Q\left(p_{I}\right) \prod_{j=1}^{N} \mathcal{V}_{j}^{(0)}\right\rangle d \mu_{0}[\delta] \\
\mathcal{R}_{i}[\delta]=- & \left\langle Q\left(p_{I}\right) \delta \xi^{+}\left(z_{i}\right) \epsilon_{i}^{\mu} \psi_{+}\left(z_{i}\right) e^{i k_{i} x_{+}\left(z_{i}\right)}\right. \\
& \left.\times\left(\frac{1}{2 \pi} \int \chi S \prod \mathcal{V}_{l}^{(0)}+\sum_{j \neq i} \mathcal{V}_{j}^{(1)} \prod_{l \neq i, j} \mathcal{V}^{(0)}\right)\right\rangle d \mu_{0}[\delta]
\end{aligned}
$$

under changes of Beltrami differentials by $\delta \hat{\mu}_{\bar{z}}^{z}=\partial_{\bar{z}} \delta v^{z}$ and changes of gravitino slices by $\delta \chi_{\bar{z}}{ }^{+}=-2 \partial_{\bar{z}} \delta \xi^{+}, \delta \hat{\mu}_{\bar{z}}=\delta \xi^{+} \chi_{\bar{z}}^{+}$. Note that there are no exterior derivative in the moduli variables $\Omega_{I J}$. As explained in Section $\S 2$, changes in $\mathcal{B}[\delta]$ of the above form leave the integrated amplitudes invariant.

3.5. Modular forms and $\vartheta$ constants. The chiral amplitudes $\mathcal{B}[\delta]\left(z_{i} ; k_{i}, \epsilon_{i} ; p_{I}^{\mu}\right)$ have now to be evaluated, certainly explicitly enough so that the relative phases $\epsilon_{\delta}$ can be determined, which would lead to a modular invariant integral formula for the superstring amplitude $\mathbf{A}_{I I}\left(k_{i}, \epsilon_{i}\right)$. In principle, all the correlation functions needed are of free fields, and the chiral determinants needed can be obtained from the chiral bosonization formulas of $[\mathbf{2 9}],[\mathbf{3 0}],[\mathbf{3 1}]$. However, these formulas depend typically on many extraneous points whose presence makes the modular transformations obscure. For our purposes, it is then important to completely eliminate these points, and remarkably, this turns out to be possible.

- We begin with the evaluation of the 0-point function, or equivalently, of $d \mu_{2}[\delta]$. Here we exploit the independence of the expression (2.17) to work in the split gauge, where the points $q_{1}, q_{2}$ are chosen to satisfy the $\delta$ dependent relation

$$
S_{\delta}\left(q_{1}, q_{2}\right)=0 .
$$

All dependence on $p_{a}, q_{\alpha}$ then manifestly cancels out, and we obtain the expression

$$
d \mu_{2}[\delta]=\prod_{I \leq J} d \Omega_{I J} \vartheta[\delta]^{4} \frac{\left\langle\nu_{1} \mid \nu_{2}\right\rangle \mathcal{M}_{\nu_{1} \nu_{2}}+\left\langle\nu_{2} \mid \nu_{3}\right\rangle \mathcal{M}_{\nu_{2} \nu_{3}}+\left\langle\nu_{3} \mid \nu_{1}\right\rangle \mathcal{M}_{\nu_{3} \nu_{1}}}{16 \pi^{2} \mathcal{M}_{\nu_{1} \nu_{2}}^{2} \mathcal{M}_{\nu_{2} \nu_{3}}^{2} \mathcal{M}_{\nu_{3} \nu_{1}}^{2}},
$$

where the bilinear $\vartheta$-constant $\mathcal{M}_{\nu_{i} \nu_{j}}$ is defined by

$$
\mathcal{M}_{\nu_{i} \nu_{j}}=\partial_{1} \vartheta\left[\nu_{i}\right] \partial_{2} \vartheta\left[\nu_{j}\right]-\partial_{2} \vartheta\left[\nu_{i}\right] \partial_{1} \vartheta\left[\nu_{j}\right] .
$$


In general, derivatives of $\vartheta$ functions do not transform well under modular transformations. However, the following identity overcomes this difficulty and leads to the expression (2.25) announced earlier for $d \mu_{2}[\delta]$

$$
\mathcal{M}_{\nu_{1} \nu_{2}}^{2}=\pi^{4} \vartheta[\delta]^{2} \prod_{k=3,4,5} \vartheta\left[\nu_{1}+\nu_{2}+\nu_{k}\right]^{2} .
$$

- To evaluate the $N$-point function, we need to evaluate the contributions of the vertex operators as well as of the component $d \mu_{0}[\delta]$ of the string chiral measure. Since the relative phases $\epsilon_{\delta}$ of the GSO projection have been already determined to be 1 , it suffices to consider the sum over spin structures $\delta$ of these contributions with these phases. In this case, clearly the split gauge is not appropriate since it is $\delta$-dependent. Instead, we shall work in the unitary gauge, where the points $q_{\alpha}$ are chosen to be the zeroes of a fixed holomorphic $(1,0)$-form $\varpi(z)$

$$
\varpi\left(q_{1}\right)=0, \quad \varpi\left(q_{2}\right)=0 .
$$

This gauge has the very important property that there exists a singlevalued scalar function $\Lambda(z)$ satisfying

$$
\begin{aligned}
\hat{\mu}_{\bar{z}}{ }^{z} & =S_{\delta}\left(q_{1}, q_{2}\right) \mu(z) \\
\mu(z) \varpi(z) & =\partial_{\bar{z}} \Lambda(z) .
\end{aligned}
$$

We then need many $\vartheta$ function identities, of which the most difficult are perhaps the ones involving the fermion stress tensor, and hence the term

$$
\varphi[\delta]\left(w ; z_{1}, z_{2}\right)=S_{\delta}\left(z_{1}, w\right) \partial_{w} S_{\delta}\left(w, z_{2}\right)-S_{\delta}\left(z_{2}, w\right) \partial_{w} S_{\delta}\left(w, z_{1}\right)
$$

For the $N$-point function with $N \leq 3$, the existence of the function $\Lambda(z)$ turns out to imply the integral identities

$$
\begin{aligned}
& \int \mu(w) I_{13}\left(w ; z_{1}, z_{2}\right)=0 \\
& \int \mu(w) \varpi(w)\left\{I_{14}\left(w ; z_{1}, z_{2}, z_{3}\right)\right. \\
& \left.\quad+I_{14}\left(w ; z_{2}, z_{3}, z_{1}\right)+I_{14}\left(w ; z_{3}, z_{1}, z_{2}\right)\right\}=0
\end{aligned}
$$

where the expressions $I_{13}\left(w ; z_{1}, z_{2}\right)$ and $I_{14}\left(w ; z_{1}, z_{2}, z_{3}\right)$ are defined by (3.46)

$$
\begin{aligned}
I_{13}\left(w ; z_{1}, z_{2}\right) & =\sum_{\delta} \mathcal{Z}[\delta] S_{\delta}\left(q_{1}, q_{2}\right) \varphi[\delta]\left(w ; z_{1}, z_{2}\right) S_{\delta}\left(z_{2}, z_{1}\right) \\
I_{14}\left(w ; z_{1}, z_{2}, z_{3}\right) & =\sum_{\delta} \mathcal{Z}[\delta] S_{\delta}\left(q_{1}, q_{2}\right) \varphi[\delta]\left(w ; z_{1}, z_{2}\right) S_{\delta}\left(z_{2}, z_{3}\right) S_{\delta}\left(z_{3}, z_{1}\right) .
\end{aligned}
$$

These identities imply in turn that $\sum_{\delta} \mathcal{B}[\delta]=0$ for $N \leq 3$.

- The 4-point function is considerably more complicated, since we need to extract Dolbeault exact differentials from $\sum_{\delta} \mathcal{B}[\delta]$ before we can arrive at a holomorphic and gauge-independent form $\mathcal{H}$. Also, we 
need identities of two types, those involving sums with $\mathcal{Z}[\delta]$, and those involving sums with $\Xi_{6}[\delta]$. We illustrate these identities with some examples. Consider first the sums involving $\mathcal{Z}[\delta]$ :

$$
\begin{aligned}
& I_{15}\left(w ; z_{1}, z_{2}, z_{3}, z_{4}\right) \\
& =\sum_{\delta} \mathcal{Z}[\delta] S_{\delta}\left(q_{1}, q_{2}\right) \varphi[\delta]\left(w ; z_{1}, z_{2}\right) S_{\delta}\left(z_{2}, z_{3}\right) S_{\delta}\left(z_{3}, z_{4}\right) S_{\delta}\left(z_{4}, z_{1}\right) \\
& I_{16}\left(w ; z_{1}, z_{2}, z_{3}, z_{4}\right) \\
& =\sum_{\delta} \mathcal{Z}[\delta] S_{\delta}\left(q_{1}, q_{2}\right) \varphi[\delta]\left(w ; z_{1}, z_{2}\right) S_{\delta}\left(z_{2}, z_{1}\right) S_{\delta}\left(z_{3}, z_{4}\right)^{2}, \\
& I_{15}^{S}\left(w ; z_{1}, z_{2}, z_{3}, z_{4}\right) \\
& =\frac{1}{2}\left(I_{15}\left(w ; z_{1}, z_{2}, z_{3}, z_{4}\right)+I_{15}\left(w ; z_{2}, z_{1}, z_{3}, z_{4}\right)\right) \\
& I_{15}^{A}\left(w ; z_{1}, z_{2}, z_{3}, z_{4}\right) \\
& =\frac{1}{2}\left(I_{15}\left(w ; z_{1}, z_{2}, z_{3}, z_{4}\right)-I_{15}\left(w ; z_{2}, z_{1}, z_{3}, z_{4}\right)\right),
\end{aligned}
$$

their integrated versions,

$$
\begin{aligned}
\mathcal{I}_{15}\left(z_{1}, z_{2}, z_{3}, z_{4}\right) & =\frac{1}{2 \pi} \int \mu(w) I_{15}\left(w ; z_{1}, z_{2}, z_{3}, z_{4}\right) \\
\mathcal{I}_{16} & =\frac{1}{2 \pi} \int \mu(w) I_{15}\left(w ; z_{1}, z_{2}, z_{3}, z_{4}\right),
\end{aligned}
$$

the following cyclically permuted integrated versions,

$$
\begin{aligned}
\mathcal{I}_{15}^{C}\left(z_{1}, z_{2}, z_{3}, z_{4}\right)= & +\mathcal{I}_{15}\left(z_{1}, z_{2}, z_{3}, z_{4}\right)+\mathcal{I}_{15}\left(z_{2}, z_{3}, z_{4}, z_{1}\right) \\
& +\mathcal{I}_{15}\left(z_{3}, z_{4}, z_{1}, z_{2}\right)+\mathcal{I}_{15}\left(z_{4}, z_{1}, z_{2}, z_{3}\right) \\
\mathcal{I}_{16}^{C}\left(z_{1}, z_{2} ; z_{3}, z_{4}\right)= & +\mathcal{I}_{16}\left(z_{1}, z_{2}, z_{3}, z_{4}\right)+\mathcal{I}_{16}\left(z_{3}, z_{4}, z_{1}, z_{2}\right),
\end{aligned}
$$

their symmetrized versions,

$$
\begin{aligned}
3 \mathcal{I}_{15}^{S}\left(z_{1}, z_{2}, z_{3}, z_{4}\right)= & \mathcal{I}_{15}^{C}\left(z_{1}, z_{2}, z_{3}, z_{4}\right)+\mathcal{I}_{15}^{C}\left(z_{1}, z_{3}, z_{4}, z_{2}\right) \\
& +\mathcal{I}_{15}^{C}\left(z_{1}, z_{4}, z_{2}, z_{3}\right) \\
3 \mathcal{I}_{16}^{S}\left(z_{1}, z_{2}, z_{3}, z_{4}\right)= & \mathcal{I}_{16}^{C}\left(z_{1}, z_{2} ; z_{3}, z_{4}\right)+\mathcal{I}_{16}^{C}\left(z_{1}, z_{3} ; z_{4}, z_{2}\right) \\
& +\mathcal{I}_{16}^{C}\left(z_{1}, z_{4} ; z_{2}, z_{3}\right),
\end{aligned}
$$

and their anti-symmetrized versions,

$$
\begin{aligned}
& 3 \mathcal{I}_{15}^{A}\left(z_{1}, z_{4} \mid z_{2}, z_{3}\right)=\mathcal{I}_{15}^{C}\left(z_{1}, z_{2}, z_{3}, z_{4}\right)-\mathcal{I}_{15}^{C}\left(z_{1}, z_{3}, z_{2}, z_{4}\right) \\
& 3 \mathcal{I}_{16}^{A}\left(z_{1}, z_{4} \mid z_{2}, z_{3}\right)=\mathcal{I}_{16}^{C}\left(z_{1}, z_{2} ; z_{3}, z_{4}\right)-\mathcal{I}_{16}^{C}\left(z_{1}, z_{3} ; z_{2}, z_{4}\right) .
\end{aligned}
$$


Then we have the following identities

$$
\begin{aligned}
& \mathcal{I}_{15}^{S}\left(z_{1}, z_{2}, z_{3}, z_{4}\right)=-2 \mathcal{I}_{16}^{S}\left(z_{1}, z_{2}, z_{3}, z_{4}\right)=-4 \mathcal{Z}_{0} \sum_{i=1}^{4} \partial \Lambda\left(z_{i}\right) \prod_{j \neq i} \varpi\left(z_{j}\right) \\
& \mathcal{I}_{15}^{A}\left(z_{1}, z_{4} \mid z_{2}, z_{3}\right)=-\mathcal{I}_{16}^{A}\left(z_{1}, z_{4} \mid z_{2}, z_{3}\right)=\frac{\zeta^{1} \zeta^{2}}{4 \pi^{2}} \Delta\left(z_{1}, z_{4}\right) \Delta\left(z_{2}, z_{3}\right),
\end{aligned}
$$

where $\Delta(z, w)$ is the bi-holomorphic form in $z, w$ introduced earlier, and $\mathcal{Z}_{0}$ is the following quantity,

$$
\mathcal{Z}_{0}=\frac{Z^{12}}{\pi^{12} \Psi_{10}(\Omega) E\left(q_{1}, q_{2}\right)^{2} \sigma\left(q_{1}\right)^{2} \sigma\left(q_{2}\right)^{2}},
$$

with $Z$ the partition function of a single chiral boson, expressible in terms of arbitrary points $r_{1}, r_{2}, r_{3}$,

$$
Z^{3}=\frac{\vartheta\left(r_{1}+r_{2}-r_{3}-\Delta\right) E\left(r_{1}, r_{2}\right) \sigma\left(r_{1}\right) \sigma\left(r_{2}\right)}{E\left(r_{1}, r_{3}\right) E\left(r_{2}, r_{3}\right) \sigma\left(r_{3}\right) \operatorname{det} \omega_{I}\left(r_{j}\right)} .
$$

Next, consider the sums involving $\Xi_{6}[\delta](\Omega)$

$$
\begin{aligned}
& I_{20}\left(z_{1}, z_{2} ; z_{3}, z_{4}\right)=\sum_{\delta} \Xi_{6}[\delta] \vartheta[\delta]^{4} S_{\delta}\left(z_{1}, z_{2}\right)^{2} S_{\delta}\left(z_{3}, z_{4}\right)^{2} \\
& I_{21}\left(z_{1}, z_{2}, z_{3}, z_{4}\right)=\sum_{\delta} \Xi_{6}[\delta] \vartheta[\delta]^{4} S_{\delta}\left(z_{1}, z_{2}\right) S_{\delta}\left(z_{2}, z_{3}\right) S_{\delta}\left(z_{3}, z_{4}\right) S_{\delta}\left(z_{4}, z_{1}\right) .
\end{aligned}
$$

Then we have the identities

$$
\begin{aligned}
& I_{20}\left(z_{1}, z_{2} ; z_{3}, z_{4}\right)=-4 \pi^{4} \Psi_{10}(\Omega)\left(\Delta\left(z_{1}, z_{3}\right) \Delta\left(z_{2}, z_{4}\right)+\Delta\left(z_{1}, z_{4}\right) \Delta\left(z_{2}, z_{3}\right)\right. \\
& I_{21}\left(z_{1}, z_{2}, z_{3}, z_{4}\right)=4 \pi^{4} \Psi_{10}(\Omega)\left(\Delta\left(z_{1}, z_{2}\right) \Delta\left(z_{3}, z_{4}\right)-\Delta\left(z_{1}, z_{4}\right) \Delta\left(z_{2}, z_{3}\right)\right.
\end{aligned}
$$

as well as the identity

$$
\sum_{I J K L} \omega_{I}\left(z_{1}\right) \omega_{J}\left(z_{2}\right) \omega_{K}\left(z_{3}\right) \omega_{L}\left(z_{4}\right) \sum_{\delta} \Xi_{6}[\delta] \vartheta[\delta]^{3} \partial_{I} \partial_{J} \partial_{K} \partial_{L} \vartheta[\delta](0)=0 .
$$

All these identities combine to give the desired formulas for $\sum_{\delta} \mathcal{B}[\delta]\left(z_{i}\right.$; $\left.k_{i}, \epsilon_{i} ; p_{I}^{\mu}\right)$ and $\mathcal{H}\left(z_{i} ; k_{i}, \epsilon_{i} ; p_{I}^{\mu}\right)$. A crucial phenomenon is that all effects of gauge choices reside only in the exact differentials $\mathcal{R}\left(z_{i} ; k_{i}, \epsilon_{i} ; p_{I}^{\mu}\right)$ which drops out of the final physical amplitude $\mathbf{A}_{I I}\left(k_{i}, \epsilon_{i}\right)$, and that $\mathcal{H}\left(z_{i} ; k_{i}, \epsilon_{i} ; p_{I}^{\mu}\right)$ is completely gauge independent.

3.6. Proof of non-renormalization theorems. To obtain scattering amplitudes in the heterotic string, we combine the anti-holomorphic factors from the type II superstring amplitudes with the holomorphic factors from the 10-dimensional bosonic string and internal 
fermions. The correlation functions can be evaluated in a straightforward manner. The main issue in the non-renormalization theorems is whether the poles in the Mandelstam variables $s_{i j}=-2 k_{i} \cdot k_{j}$ from the holomorphic sector survive after combination with the anti-holomorphic sector and integration on the worldsheet. The most difficult amplitudes are the $R^{2} F^{2}$ and the $R^{4}$ amplitudes, so we discuss them briefly. with

For $R^{2} F^{2}$, the holomorphic sector is $\mathcal{W}=\mathcal{W}_{\left(R^{2}\right)}\left(z_{1}, z_{2}\right) \mathcal{W}_{\left(F^{2}\right)}\left(z_{3}, z_{4}\right)$,

$$
\begin{aligned}
\mathcal{W}_{\left(R^{2}\right)}=\left(\epsilon_{1} \cdot \epsilon_{2}\right) & \partial_{z_{1}} \partial_{z_{2}} G\left(z_{1}, z_{2}\right) \\
& -\sum_{i j}\left(\epsilon \cdot k_{i}\right)\left(\epsilon \cdot k_{j}\right) \partial_{z_{1}} G\left(z_{1}, z_{j}\right) \partial_{z_{2}} G\left(z_{2}, z_{j}\right) .
\end{aligned}
$$

This term leads to poles in $s_{i j}$. However, up to total derivatives on $\Sigma$, $s_{i j} \mathcal{W}_{\left(R^{2}\right)}$ can be replaced by expressions of the form

$$
\begin{aligned}
s_{12} \mathcal{W}_{\left(R^{2}\right)} \rightarrow 2\left(f_{1} f_{2}\right) \partial_{z_{1}} \partial_{z_{2}} G\left(z_{1}, z_{2}\right) & \\
& -2 \sum_{i j} k_{i}^{\mu} f_{1}^{\mu \nu} f_{2}^{\nu \rho} k_{j}^{\rho} \partial_{z_{1}} G\left(z_{1}, z_{i}\right) \partial_{z_{2}} G\left(z_{2}, z_{j}\right)
\end{aligned}
$$

Since the anti-holomorphic sector $\overline{\mathcal{Y}_{S}}$ always includes an $s_{i j}$ factor, and since the above right hand side integrates to 0 against anti-holomorphic forms, these amplitudes do not contribute to the low-energy effective action.

For $R^{4}$, the holomorphic sector is given by the expression $\mathcal{W}=$ $\mathcal{W}_{\left(R^{4}\right)}$ in (2.36). In this case, only the expressions $s_{i j} s_{k l} \mathcal{W}_{\left(R^{4}\right)}$ can be replaced, up to total derivatives and to terms which vanish when $s_{i j} \rightarrow$ 0 , by sum of regular expressions tending to 0 as $s_{i j} \rightarrow 0$. However, when we expand the exponential factor $\exp \left(\sum_{i j} s_{i j} G\left(z_{i}, z_{j}\right)\right)$ at low energy, we find that the contributions of the constant term integrate to 0 . Thus we need only consider the terms from the exponential factor with at least one power of $s_{i j}$. Combined with the other factor $s_{k l}$ from $\overline{\mathcal{Y}_{S}}$, we can apply then the previous result for $s_{i j} s_{k l} \mathcal{W}_{\left(R^{4}\right)}$, and obtain the desired non-renormalization theorem.

3.7. S-duality and factorization. The expressions (2.33) determine the superstring scattering amplitudes only up to a constant factor depending only on the topology of the worldsheet. This constant factor $\mathrm{C}_{2}$ should be determined ultimately by the factorization properties of the physical amplitudes. To compare with the S-duality predictions for the two-loop correction to the $D^{4} R^{4}$ term in the effective action, we need to compare two non-vanishing quantities, and the above constant factor has to be determined precisely. For this, we have to analyze the contributions in $\mathbf{A}_{I I}\left(k_{i}, \epsilon_{i}\right)$ of the region of the moduli space $\mathcal{M}_{2}$ near the divisor of separating nodes, and identify the resulting pole in $s \equiv s_{12}$ 
at $s=\frac{4}{\alpha^{\prime}}$. Restoring the string tension parameter $\alpha^{\prime}$, the coupling constant $\lambda$, and the normalization $\kappa$ for the massless vertex operators, we can write the amplitude $\mathbf{A}_{I I}$ as

$$
\begin{aligned}
\mathbf{A}_{I I}\left(k_{i}, \epsilon_{i}\right)=C_{2} e^{2 \lambda} & K \bar{K} \kappa^{4} \int_{\mathcal{M}_{2}} \frac{\left|\prod_{I \leq J} d \Omega_{I J}\right|^{2}}{(\operatorname{det} \operatorname{Im} \Omega)^{5}} \\
& \times \int_{\Sigma^{4}}\left|\mathcal{Y}_{S}\right|^{2} \exp \left(-\frac{\alpha^{\prime}}{2} \sum_{i<j} k_{i} \cdot k_{j} G\left(z_{i}, z_{j}\right)\right)
\end{aligned}
$$

and we find $[\mathbf{1 4}]$

$$
\mathcal{A}_{I I}=-\delta(k) \frac{2^{6} \pi^{3} C_{2} / \alpha^{\prime}}{s-4 / \alpha^{\prime}} e^{2 \lambda} K \bar{K} \mathcal{B}_{1}^{(3)}\left(k_{1}, k_{2},-q\right) \mathcal{B}_{1}^{(3)}\left(k_{3}, k_{4}, q\right),
$$

where $\mathcal{B}_{1}^{(3)}\left(k_{1}, k_{2}, q\right)$ and $\mathcal{B}_{1}^{(3)}\left(k_{3}, k_{4},-q\right)$ are one-loop 3-point functions given by

$$
\begin{aligned}
\mathcal{B}_{1}^{(3)}\left(k_{1}, k_{2},-q\right)= & \int_{\mathcal{M}_{1}} \frac{\left|d \tau_{11}\right|}{\left|I m \tau_{11}\right|^{5}} \int d^{2} z_{1} d^{2} z_{2} \exp \frac{\alpha^{\prime} s}{4}\left\{G\left(z_{1}, z_{2}\right)\right. \\
& \left.-G\left(z_{1}, p_{1}\right)-G\left(z_{2}, p_{1}\right)\right\} \\
\mathcal{B}_{1}^{(3)}\left(k_{3}, k_{4}, q\right)= & \int_{\mathcal{M}_{1}} \frac{\left|d \tau_{22}\right|}{\left|I m \tau_{22}\right|^{5}} \int d^{2} z_{3} d^{2} z_{4} \exp \frac{\alpha^{\prime} s}{4}\left\{G\left(z_{3}, z_{4}\right)\right. \\
& \left.-G\left(z_{3}, p_{2}\right)-G\left(z_{4}, p_{2}\right)\right\} .
\end{aligned}
$$

Comparing this with the factorization of tree-level and one-loop amplitudes, we obtain the desired constant $C_{2}$. With the normalization for the tree-level 4-point function given in $[\mathbf{1 4}]$, we have $C_{2}=\frac{\sqrt{2}}{2^{6}\left(\alpha^{\prime}\right)^{5}}$.

Taking the limit $k_{i} \rightarrow 0$ in $A_{I I}$, the low-energy two-loop contribution to the $D^{4} R^{4}$ is then found to be

$$
A_{2}^{\left(D^{4} R^{4}\right)}=8 V_{2} C_{2} e^{2 \lambda}\left(\alpha^{\prime}\right)^{2}\left(s^{2}+t^{2}+u^{2}\right) \kappa^{4} K \bar{K},
$$

where $V_{2}$ is the volume of the fundamental domain of $\operatorname{Sp}(4, \mathbf{Z}) / \mathbf{Z}_{2}$. This volume has been determined by Siegel $[\mathbf{4 1}]$, and combined with the value for $C_{2}$ just found, we find complete agreement with S-duality.

3.8. Orbifolds and KKS models. The difficulties with gaugefixing superstring amplitudes reside only with the superghost part of the theory. For more general space-times, the same method applies and gives well-behaved amplitudes, as long as the earlier matter part $x^{\mu}, \psi_{ \pm}^{\mu}$ is replaced by a compactification which respects world-sheet supersymmetry.

For $\mathbf{Z}_{2}$ orbifold models, the essential new features are the twisted bosonic propagator $B_{\epsilon}(z, w)=\left\langle\partial_{z} x(w) \partial_{w} x(w)\right\rangle_{\epsilon}$, and the supersymmetric extension of the Prym period matrix. The first is found to be

$$
B_{\epsilon}(z, w)=S_{\delta_{i}^{+}}(z, w) S_{\delta_{i}^{-}}(z, w)+b_{i} \omega_{\epsilon}(z) \omega_{\epsilon}(w)
$$


where $\omega_{\epsilon}(z)$ is the Prym differential. On the other hand, there are subtleties with the second: while the supersymmetric extension $\hat{\Omega}_{I J}$ can be identified both as period matrix of a new complex structure $\hat{g}_{m n}$ and as covariance matrix of the chirally split amplitudes [28], the Prym matrix $\hat{\tau}_{\epsilon}$ of $\hat{g}_{m n}$ and the covariance $\tilde{\tau}_{\epsilon}$ of the chirally split twisted amplitudes are distinct supersymmetric extensions of the Prym matrix $\tau_{\epsilon}$. Their difference is

$$
\begin{aligned}
\Delta \tau_{\epsilon}=-\frac{i}{8 \pi} \iint d^{2} z d^{2} w \chi_{\bar{z}}{ }^{+} S_{\delta}(z, w) \chi_{\bar{w}}{ }^{+} & \\
\times & \left\{\omega_{\epsilon}(z) \omega_{\epsilon}(w)-\omega_{I}(z) \omega_{J}(w) \frac{\partial \hat{\tau}_{\epsilon}}{\partial \hat{\Omega}_{I J}}\right\},
\end{aligned}
$$

where $\hat{\tau}$ is viewed as a function of $\hat{\Omega}_{I J}$. Only after taking properly into account such corrections can we arrive at the correct $\mathbf{Z}_{2}$ gauge-fixed orbifold measure.

\section{Directions for Further Investigation}

In this section, we discuss a number of directions for possible further investigation.

4.1. Higher genus superstrings. The solution of two-loop superstrings gives us some optimism for an eventual complete solution of superstring perturbation theory. Nevertheless, the two-loop case benefits of a number of simplifying features: the $\bar{\partial}$ operator is always invertible for even spin structures $\delta$, the super period matrix $\hat{\Omega}_{I J}$ of a supergeometry is always defined (instead of away from a subvariety), and we can construct explicitly the fiber of supermoduli space over a fixed $\hat{\Omega}_{I J}$. In the bosonic string at 3-loops, it has been pointed out that the spurious poles in the bosonic string integrand resulting from the $\vartheta$ divisor can be cancelled by the zeroes from the measure $\prod_{I \leq J} d \Omega_{I J}$ [42]. We can hope that a similar mechanism will take place for the superstring. However, a manageable construction of the fibers remains a challenging problem, and clearly much work will be needed.

Alternatively, we can look for Ansätze for the 3-loop superstring measure from factorization constraints, now that the 2-loop measure is known. For example, if in analogy with the 2-loop case, we take as Ansätz for the 3-loop string measure an expression of the form

$$
d \mu[\Delta]\left(\Omega^{(3)}\right)=\frac{\vartheta[\Delta]\left(\Omega^{(3)}\right)^{4} \Xi_{6}[\Delta]\left(\Omega^{(3)}\right)}{8 \pi^{4} \Psi_{9}\left(\Omega^{(3)}\right)} \prod_{I \leq J} d \Omega_{I J}^{(3)},
$$

where $\Psi_{9}\left(\Omega^{(3)}\right)^{2}=\prod_{\Delta \text { even }} \vartheta[\Delta]\left(\Omega^{(3)}\right)$ is Igusa's modular form [43], then $\Xi_{6}[\Delta]\left(\Omega^{(3)}\right)$ must be a modular covariant form of weight 6 satisfying 
the factorization constraint

$$
\lim _{t \rightarrow 0} \Xi_{6}[\Delta]\left(\Omega^{(3)}\right)=\eta\left(\Omega^{(1)}\right)^{12} \Xi_{6}[\delta]\left(\Omega^{(2)}\right)
$$

in the limit where the genus 3 surface with period matrices degenerates into surfaces of genera 1 and 2 with period matrices $\Omega^{(1)}$ and $\Omega^{(2)}$, and the genus 3 spin structure $\Delta$ factors into two even spin structures. Polynomials in $\vartheta$ constants have been found which can be candidates for $\Xi_{6}[\Delta]\left(\Omega^{(3)}\right)^{2}$. It may be valuable to pursue this further [44].

In another direction, we may try to generalize directly the higher genus 4-point function from the very simple final expression (2.33) for genus 2 and factorization properties. Several candidates have now been proposed along these lines $[\mathbf{4 5}]$.

4.2. Odd spin structures. For $N$ sufficiently large, the odd spin structures of the worldsheet $\Sigma$ will begin contributing to the $N$-point function. It would be important to extend our gauge-fixing method to this case as well. The chiral splitting of the matter fields $x^{\mu}, \psi_{ \pm}^{\mu}$ has been carried out in $[\mathbf{2 8}]$ for odd spin structures $\delta$. A new phenomenon is the emergence of an additional superholomorphic form $\hat{\omega}_{0}$ which is the supersymmetric extension of the holomorphic form $h_{\delta}(z)$ on $\kappa_{\delta}^{1 / 2}(\Sigma)$. This is a source of new difficulties, since from a certain point of view, the analogue of the super period matrix is now $(h+1) \times(h+1)$ dimensional.

4.3. BRST formalism. The BRST symmetry is a powerful symmetry of gauge fixed quantum field theories and particularly of string theories. Higher loop superstring amplitudes based on BRST symmetry have been proposed a long time ago by Friedan, Martinec, and Shenker [3]. However, the BRST invariance guarantees the gauge slice independence of these amplitudes only up to total derivatives on local patches on moduli space [11]. In retrospect, we see that the gauge-fixing method based on super period matrices has produced both local and global corrections to the BRST prescription, under the form of an insertion of the stress tensor and of the finite-dimensional determinants in (2.9). It may be valuable to re-examine the amplitudes in this light, and determine whether they can be arrived at by a BRST-like prescription. The BRST formalism has also been re-examined for the bosonic string in [46], from other considerations.

4.4. Effective actions. As we had mentioned earlier, the two-loop amplitudes allow us to determine the two-loop corrections to the effective action, and as a by-product, to get an indirect check of the many dualities conjectured in string theory $[\mathbf{4 7}, \mathbf{4 8}, 49]$. The consistency with the conjectured S-duality of the type IIB superstring has now been checked. However, the relation with dualities of the non-renormalization of the $\mathcal{R}^{4}$ term and the correction to the $D^{2} F^{4}$ term in the heterotic string is still obscure (see $[\mathbf{1 4}]$ and references therein). 
4.5. Normalizations of determinants. The bosonization formulas of Fay [30], Faltings [31], and Verlinde-Verlinde [29] determine the chiral determinants of $\bar{\partial}$ operators up to constants depending only on the genus. The exact value of these constants for the $\bar{\partial}$ operator on scalars has received significant attention over the years [50]. It would be useful to determine them for the $\bar{\partial}$ operator for all weights.

\section{References}

[1] M. Green and J. Schwarz, Supersymmetrical string theories, Phys. Lett. 109 B (1982) 444-448.

[2] D.J. Gross, J.A. Harvey, E.J. Martinec, and R. Rohm, Heterotic String Theory. 1. The Free Heterotic String, Nucl. Phys. B 256253 (1985); Heterotic String Theory. 2. The Interacting Heterotic String, Nucl. Phys. B 267 (1986) 75.

[3] D. Friedan, E. Martinec, and S. Shenker, Conformal invariance, supersymmetry, and string theory, Nucl. Phys. B 271 (1986) 93.

[4] E. D'Hoker and D.H. Phong, Loop amplitudes for the fermionic string, Nucl. Phys. B 278 (1986) 225;

G. Moore, P. Nelson, and J. Polchinski, Strings and supermoduli, Phys. Lett. B 169 (1986) 47-53.

[5] E. D'Hoker and D.H. Phong, Two-Loop Superstrings I, Main Formulas, Phys. Lett. B529 (2002) 241-255; hep-th/0110247;

E. D'Hoker and D.H. Phong, Lectures on two loop superstrings, hep-th/0211111.

[6] E. D'Hoker and D.H. Phong, Two-Loop Superstrings II, The chiral Measure on Moduli Space, Nucl. Phys. B636 (2002) 3-60; hep-th/0110283.

[7] E. D'Hoker and D.H. Phong, Two-Loop Superstrings III, Slice Independence and Absence of Ambiguities, Nucl. Phys. B636 (2002) 61-79; hep-th/0111016.

[8] E. D'Hoker and D.H. Phong, Two-Loop Superstrings IV, The Cosmological Constant and Modular Forms, Nucl. Phys. B639 (2002) 129-181; hep-th/0111040.

[9] E. D'Hoker and D.H. Phong, Two-Loop Superstrings V, Gauge Slice Independence of the N-Point Function, Nucl. Phys. B715 (2005) 91-119; hepth/0501196.

[10] E. D'Hoker and D.H. Phong, Two-loop Superstrings VI, Non-renormalization theorems and the 4-Point function, Nucl. Phys. B715 (2005) 3-90; hepth/0501197.

[11] E. Verlinde and H. Verlinde, Multiloop calculations in covariant superstring theory, Phys. Lett. B192 (1987) 95-102;

$\mathrm{H}$. Verlinde, A note on the integral over fermionic supermoduli, Utrecht Preprint No. THU-87/26 (1987) unpublished.

[12] J. Atick, J. Rabin, and A. Sen, An ambiguity in fermionic string theory, Nucl. Phys. B 299 (1988) 279-294;

G. Moore and A. Morozov, Some remarks on two-loop string calculations, Nucl. Phys. B 306 (1988) 387-404;

J. Atick, G. Moore, and A. Sen, Some global issues in string perturbation theory, Nucl. Phys. B 308 (1988) 1; Catoptric tadpoles, Nucl. Phys. B 307 (1988) 221273 ;

H. La and P. Nelson, Unambiguous fermionic string amplitudes, Phys. Rev. Lett. 63 (1989) 24-27.

[13] E. Martinec, Non-renormalization Theorems and Fermionic String Finiteness, Phys. Lett. B171 (1986) 189;

[14] E. D'Hoker, M. Gutperle, and D.H. Phong, Two-loop superstrings and S-duality, Nucl.Phys. B 722 (2005) 81-118, hep-th/0503180. 
[15] S. Kachru, J. Kumar, and E. Silverstein, Vacuum energy cancellation in a nonsupersymmetric string, Phys. Rev. D 59 (1999) 106004, hep-th 9807076;

S. Kachru and E. Silverstein, Self-dual nonsupersymmetric type II string compactifications, JHEP 9811 (1998) 001, hep-th 9808056;

S. Kachru and E. Silverstein, On vanishing two-loop cosmological constants in nonsupersymmetric strings, JHEP 9901 (1999) 004, hepth 9810129.

[16] K. Aoki, E. D'Hoker, and D.H. Phong, Two-loop superstrings on orbifold compactifications, Nucl. Phys. 688 (2004) 3-69, hep-th/0312181;

K. Aoki, E. D'Hoker, and D.H. Phong, On the construction of asymmetric orbifold models, Nucl.Phys. B 695 (2004) 132-168, hep-th/0402134.

[17] S. Mandelstam, Interacting string picture of the fermionic string, in 'Workshop on Unified String Theories', eds. M. Green and D. Gross, 1986, World Scientific, 577 ;

N. Berkovits, Calculation Of Scattering Amplitudes For The Neveu-Schwarz Model Using Supersheet Functional Integration, Nucl. Phys. B 276650 (1986);

S. Mandelstam, The $n$ loop string amplitude: Explicit formulas, finiteness and absence of ambiguities, Phys. Lett. B 27782 (1992).

[18] L. Alvarez-Gaumé, C. Gomez, G. Moore, P. Nelson, and C. Vafa, Fermionic strings in the operator formalism, Nucl. Phys. B 311 (1988) 333;

A. Neveu and P. West, Group theoretic approach to the superstring and its supermoduli, Nucl. Phys. B 311 (1988) 79.

P. Di Vecchia, K. Hornfeck, M. Frau, A. Lerda, S. Sciuto, $N$ string, G loop vertex for the fermionic string, Phys. Lett. B211 (1988) 301;

O. Yasuda, Multiloop Modular Invariance of D = 10 Type II Superstring Theory, Nucl. Phys. B318 (1989) 397.

[19] E. Gava and R. Iengo, Modular Invariance and the Two Loop Vanishing of the Cosmological Constant, Phys. Lett. B 207 (1988) 283;

R. Iengo and C.J. Zhu, Notes on Non-renormalization Theorem in Superstring Theories, Phys. Lett. B 212 (1988) 309;

A. Morozov, Pointwise Vanishing of Two-Loop Contributions to 1, 2, 3 Point Functions in Superstring Theories, Nucl. Phys. B 318 (1989) 137.

[20] A. Morozov, On the two-loop contribution to the superstring four-point function, Phys. Lett. B 209 (1988) 473-476;

O. Yasuda, Factorization of a two loop Four Point Superstring Amplitude, Phys. Rev. Lett. 60 (1988) 1688; erratum-ibid 61 (1988) 1678;

R. Iengo and C.J. Zhu, Two-loop computation of the four-particle in heterotic string theory, Phys. Lett. B 212 (1988) 313.

[21] O. Lechtenfeld and A. Parkes, On the Vanishing of the genus 2 Superstring Vacuum Amplitude, Phys. Lett. B 202 (1988) 75;

O. Lechtenfeld, On Finiteness of the Superstring, Nucl. Phys. B 322 (1989) 82;

O. Lechtenfeld and A. Parkes, On covariant multiloop superstring amplitudes, Nucl. Phys. B 332 (1990) 39-82;

O. Lechtenfeld, Factorization and modular invariance of multiloop superstring amplitudes in the unitary gauge, Nucl. Phys. B 338 (1990) 403-414.

[22] R. Iengo and C.J. Zhu, Explicit modular invariant two-loop superstring amplitude relevant to $R^{4}$, JHEP 06 (1999) 011.

[23] E. D'Hoker and D.H. Phong, The geometry of string perturbation theory, Rev. Modern Physics 60 (1988) 917-1065.

[24] G. Moore, J. Harris, P. Nelson, and I.M. Singer, Modular forms and the cosmological constant, Phys. Lett. B 178 (1986) 167-173.

[25] S.T. Yau, editor, Mathematical aspects of string theory, World Scientific, 1987.

[26] N. Berkovits, Lorentz covariant Green-Schwarz superstring amplitudes, Phys. Lett. 300 B (1993) 53, hep-th/9211025; 
N. Berkovits, Multiloop amplitudes and vanishing theorems using the pure spinor formalism for the superstring, hep-th/0406055;

N. Berkovits, Super Poincaré covariant two-loop superstring amplitudes, hepth/0503197.

[27] Y. Aisaka and Y. Kazama, Origin of pure spinor superstring, JHEP 0505, 046 (2005) [arXiv:hep-th/0502208];

Y. Aisaka and Y. Kazama, Relating Green-Schwarz and extended pure spinor formalisms by similarity transformation, JHEP 0404, 070 (2004) [arXiv:hepth/0404141].

[28] E. D'Hoker and D.H. Phong, Conformal scalar fields and chiral splitting on super Riemann surfaces, Commun. Math. Physics 125 (1989) 469-513.

[29] E. Verlinde and H. Verlinde, Chiral bosonization, determinants and the string partition function, Nucl. Phys. B288 (1987) 357.

[30] J.D. Fay, Theta Functions on Riemann Surfaces, Lecture Notes in Math., Vol 352, Springer-Verlag, Berlin, 1973.

[31] G. Faltings, Calculus on arithmetic surfaces, Ann. of Math. 119 (1984) 387-424.

[32] E. D'Hoker and D.H. Phong, Momentum analyticity, and finiteness of the oneloop superstring amplitude, Phys. Rev. Lett. 70 (1993), 3692;

E. D'Hoker and D.H. Phong, The box graph in superstring theory, Nucl. Phys. B440 (1995) 24.

E. D'Hoker and D.H. Phong, Dispersion relations in string theory, Theor. Math. Phys. 98 (1994) 306-316, Teor. Mat. Fiz. 98 (1994) 442-455, hep-th/9404128.

[33] Z.J. Zheng, J.B. Wu and C.J. Zhu, Two-loop superstrings in hyperelliptic language. I: The main results, Phys. Lett. B 559 (2003) 89, hep-th/0212191;

C.J. Zhu, Two-loop computation in superstring theory, arXiv:hep-th/0301018;

Z.J. Zheng, J.B. Wu and C.J. Zhu, Two-loop superstrings in hyperelliptic language. II: The vanishing of the cosmological constant and the nonrenormalization theorem, Nucl. Phys. B 663 (2003) 79, hep-th/0212198;

Z.J. Zheng, J.B. Wu and C.J. Zhu, Two-loop superstrings in hyperelliptic language. III: The four-particle amplitude, Nucl. Phys. B 663 (2003) 95, hepth/0212219;

J.B. Wu and C.J. Zhu, Comments on two-loop four-particle amplitude in superstring theory, JHEP 0305 (2003) 056, hep-th/0303152;

Z.G. Xiao and C.J. Zhu, Factorization and unitarity in superstring theory, JHEP 0508 (2005) 058, hep-th/0503248.

[34] D.J. Gross and E. Witten, Superstring Modifications of Einstein's Equations, Nucl. Phys. B 277 (1986), 1.

[35] M.B. Green and M. Gutperle, Effects of D-instantons, Nucl. Phys. B 498, 195 (1997) [arXiv:hep-th/9701093];

M.B. Green, M. Gutperle, and P. Vanhove, One loop in eleven dimensions, Phys. Lett. B 409, 177 (1997) hep-th/9706175.

[36] M.B. Green, H. Kwon, and P. Vanhove, Two loops in eleven dimensions, Phys. Rev. D 61 (2000) 10400, hep-th/9910055;

M.B. Green and P. Vanhove, The low energy expansion of the one-loop type II superstring amplitude, Phys. Rev. D 61 (2000) 104011, hep-th/9910056.

J.G. Russo, An ansatz for a non-perturbative four-graviton amplitude in type IIB superstring theory, Phys. Lett. B 417 (1998) 253, hep-th/9707241.

[37] M.B. Green and P. Vanhove, Duality and higher derivative terms in $M$ theory, arXiv:hep-th/0510027.

[38] P.S. Howe, Super Weyl transformations in two dimensions, J. Phys. A (1979) 393 ;

E. Martinec, Superspace geometry of superstrings, Phys. Rev. D 28 (1983) 2604;

E. D'Hoker and D.H. Phong, Superholomorphic anomalies and supermoduli 
space, Nucl. Phys. B 292 (1987) 317.

S. Giddings and P. Nelson, The geometry of super Riemann surfaces, Commun. Math. Phys. 116 (1988), 607-634.

[39] E. D'Hoker and D. H. Phong, Vertex Operators For Closed Strings, Phys. Rev. D 35, 3890 (1987).

[40] K. Aoki, E. D'Hoker and D.H. Phong, Unitarity of Closed Superstring Perturbation Theory, Nucl. Phys. B342 (1990) 149.

[41] C.L. Siegel, Symplectic geometry, Amer. J. Math. 65 (1943) 1-86.

[42] A. Belavin, V. Knizhnik, A. Morozov, and A. Perelomov, Two and three loop amplitudes in the bosonic string theory, Phys. Lett. B 177 (1986) 324-328.

[43] J.I. Igusa, Modular forms and projective invariants, Amer. J. Math. 89 (1967) $817-855$.

[44] E. D'Hoker and D.H. Phong, Asyzygies, modular forms, and the superstring measure I, Nucl. Phys. B 710 (2005) 58-82, hep-th/0411159;

E. D'Hoker and D.H. Phong, Asyzygies, modular forms, and the superstring measure II, Nucl. Phys. B 710 (2005) 83-116, hep-th/0411182.

[45] M. Matone and R. Volpato, Higher genus superstring amplitudes from the geometry of moduli space, hep-th/0506231;

C.J. Zhu, A formula for multi-loop 4-particle amplitude in superstring theory, hep-th/0503001.

[46] B. Craps and K. Skenderis, Comments on BRST quantization of strings, JHEP 0505 (2005) 001, hep-th/ 0503038.

[47] E. Witten, String theory dynamics in various dimensions, Nucl. Phys. B 443, 85 (1995) [arXiv:hep-th/9503124];

A. Dabholkar, Ten-dimensional heterotic string as a soliton, Phys. Lett. B 357, 307 (1995) [arXiv:hep-th/9506160];

C.M. Hull, String-string duality in ten-dimensions, Phys. Lett. B 357, 545 (1995) [arXiv:hep-th/9506194];

J. Polchinski and E. Witten, Evidence for Heterotic - Type I String Duality, Nucl. Phys. B 460, 525 (1996) [arXiv:hep-th/9510169].

[48] E. Kiritsis and N. A. Obers, Heterotic/type-I duality in $D<10$ dimensions, threshold corrections and D-instantons, JHEP 9710, 004 (1997) [arXiv:hepth/9709058];

C. Bachas, Heterotic versus type I, Nucl. Phys. Proc. Suppl. 68, 348 (1998) [arXiv:hep-th/9710102];

C. Bachas, C. Fabre, E. Kiritsis, N.A. Obers, and P. Vanhove, Heterotic/typeI duality and D-brane instantons, Nucl. Phys. B 509, 33 (1998) [arXiv:hepth/9707126];

M. Bianchi, Open strings and dualities, J. Korean Phys. Soc. 33 (1998) hepth/9712020;

K. Foerger and S. Stieberger, Higher derivative couplings and heterotic-type I duality in eight dimensions, Nucl. Phys. B 559, 277 (1999) [arXiv:hep-th/9901020].

[49] A.A. Tseytlin, On SO(32) heterotic - type I superstring duality in ten dimensions, Phys. Lett. B 367, 84 (1996) [arXiv:hep-th/9510173];

A.A. Tseytlin, Heterotic - type I superstring duality and low-energy effective actions, Nucl. Phys. B 467, 383 (1996) [arXiv:hep-th/9512081].

[50] P. Deligne, Le determinant de la cohomologie, Contemp. Math. 67 (1988) 93178 ;

C. Soulé, Geometrie d'Arakelov des surfaces arithmetiques, Asterisque 177-178 (1989) 327;

H. Gillet and C. Soulé, Analytic torsion and the arithmetic Todd genus, Topology 30 (1991) 21;

R. Wentworth, Asymptotics of determinants from functional integration, J. 
Math. Phys. 32 (1991) 1767-1773;

J. Jorgenson, Degenerating hyperbolic surfaces and an evaluation of the constant in Deligne's arithmetic Riemann-Roch theorem, 1991, preprint.

[51] N. Berkovits and C. Mafra, Equivalence of two-loop superstring amplitudes in the pure spinor and RNS formalisms, hep-th/0509234;

C. Mafra, Four-point one-loop amplitude computation in the pure spinor formalism, hep-th/0512052.

Department of Physics and Astronomy, University of California, Los Angeles, CA 90095

E-mail address: dhoker@physics.ucla.edu

Department of Mathematics, Columbia University, New York, NY 10027

E-mail address: phong@cpw.math.columbia.edu 